\title{
Polarization observables in vector meson photoproduction
}

\author{
Michael Pichowsky, Çetin Şavklı and Frank Tabakin \\ Department of Physics 85 Astronomy, University of Pittsburgh, Pittsburgh, Pennsylvania 15260
}

(August 15, 2018)

\begin{abstract}
The photoproduction of vector mesons $(\rho, \omega, \phi)$ is of renewed interest because intense high energy beams of polarized electrons and photons are under development. These beams and also polarized targets make it possible to explore the dynamics of basic baryon structure. As a step toward that goal, an analysis of all possible polarization observables for the case of vector meson photoproduction from a nucleon target is presented. The question of which observables are needed to determine completely the basic photoproduction amplitudes and the relationships between spin observables are addressed. Such theorems are most readily demonstrated by representing all observables as bilinear products of helicity amplitudes and using known properties of Dirac gamma and spin1 matrices. The general angular dependence of spin observables, especially near thresholds and resonances, is examined for the vector meson case. The criteria for a complete set of observables and the relationships between observables are then presented.
\end{abstract}

24.70.+s,25.20Lj,13.60Le,13.88.+e

\section{INTRODUCTION}

The photoproduction and electroproduction of mesons is of renewed interest now that CEBAF has arrived. The thresholds for $\rho, \omega$ and $\phi$ mesons will be readily attained and exceeded. The new high-intensity continuous beams, and the development of polarized targets and beams, will allow measurement of spin observables with unprecedented precision. In addition, the measurement of recoil baryons, including the $\Lambda$ (which due to its weak decay $\Lambda \rightarrow p+\pi^{-}$is spin self-analyzing), will provide even more spin observables. Vector meson decays $\left(\rho \rightarrow \pi \pi ; \omega \rightarrow \pi \pi \pi ; \phi \rightarrow K^{+} K^{-}\right)$provide a measure of their density matrices, which is equivalent to determining the intensity, polarization and tensor polarization of these vector mesons. Such measurements were made in the 1960's [1]. With CEBAF, data of higher precision and completeness should be achievable [2].

It is therefore timely and important to study the photo and electro-production of vector mesons. The associated form factors, and intermediate isobar states should test quark models. The $\phi$ meson is of particular interest because of its simple $(\bar{s} s){ }^{3} S_{1}$ - quark content. It is the positronium of strange quarks and, in parallel to charmonium $(\bar{c} c)$ states, should provide insights into basic QCD dynamics in the nonperturbative region. Perhaps an intimate connection between production of $\bar{s} s$ pairs and baryon polarization could provide detailed tests of quark descriptions. For example, the family of reactions $\gamma p \rightarrow K^{+} \Lambda, \gamma p \rightarrow K^{+} \Sigma^{0}, \bar{p} p \rightarrow \phi \phi$, and $\gamma p \rightarrow \phi p$ all involve $\bar{s} s$ strangeness production. In addition, they could also involve preexisting $\bar{s} s$ content of the initial protons and antiprotons. That possibility has been explored in a series of papers dedicated to finding direct evidence for an admixture of $\bar{s} s$ pairs in baryons [3 5 ]. Spin observable are probably most sensitive to such a configuration mixture. 
In view of the above motivation, in this paper we discuss some general features of spin observables for the photoproduction of vector mesons. Our approach is similar to that used in studies of pseudoscalar meson production and of the $\bar{p} p \rightarrow \bar{\Lambda} \Lambda$ reaction near threshold [6] 8$]$. However, for a vector meson the spin- 1 complicates the dynamics and a more general approach is required to keep track of all spin-observables and to demonstrate their general features, e.g., their nodal structure and their normal energy evolution. For example, the question of which spin observables vanish at $0^{\circ}$ and $180^{\circ}$, which have nodes, where these nodes occur and how they are expected to evolve with energy (based on simple centrifugal barrier and/or resonance constraints) are addressed here. In addition, we examine the question of what constitutes a complete set of measurements, e.g., which measurements are needed to determine the magnitude and phases of the basic amplitudes. Also, we wish to know which measurements are redundant, based on assumed symmetries. Such questions have been answered for the pseudoscalar meson case [9 11]; those discussions are extended here to the vector meson case. Indeed, generalized discussions already exist in the literature [12,13], which are however quite different from our treatment.

Our description uses a space of bilinear products of helicity amplitudes, along with well-known properties of $4 \times 4$ and $3 \times 3$ matrix bases, to reveal the general features of spin observables. We also use the idea of transversity amplitudes [14]. For clarity, we also include the pseudoscalar case using this bilinear form description and show how it generalizes to the vector meson case and indeed to many other reactions.

\section{THE BASIC AMPLITUDE AND SPIN OBSERVABLES}

For vector meson photoproduction, $\vec{\gamma}+\vec{N} \rightarrow \vec{V}+\vec{N}^{\prime}$, our basic amplitude is of the form

$$
\mathcal{F} \equiv\left\langle\vec{q} \lambda_{V} \lambda_{2}|T| \vec{k} \lambda \lambda_{1}\right\rangle,
$$

where the variables and the coordinate system conventions are presented in Fig. 1. The incident and final relative momenta are $\vec{k}$ and $\vec{q}$, respectively. Jacob \& Wick (JW) 15 phase conventions are used throughout this paper. We use $\lambda= \pm 1$ to describe the two transverse helicity states of the initial photon, $\lambda_{1}= \pm 1 / 2$ to describe the $\operatorname{target}(N)$ proton helicity, $\lambda_{V}= \pm 1,0$ for the final vector meson helicity and $\lambda_{2}$ for the final $\left(N^{\prime}\right)$ baryon helicity. For a real photon, the above amplitude represents $3 \times 2 \times 2 \times 2=24$ complex numbers. However, by virtue of parity invariance there are 12 relations between these amplitudes and consequently we have twelve independent complex helicity amplitudes or 24 real numbers, at each energy and angle. We denote these twelve helicity amplitudes by 


$$
\left\langle\vec{q} \lambda_{V} \lambda_{2}|T| \vec{p} \lambda \lambda_{1}\right\rangle \rightarrow H_{a \lambda_{V}}(\theta),
$$

where $a=1 \cdots 4$ and $\lambda_{V}= \pm 1,0$, and the particular matrix element assignments are given below. The pseudoscalar case is recovered by simply taking $\lambda_{V} \rightarrow 0$ and then we have $1 \times 2 \times 2 \times 2=8$ complex numbers and, after considering parity symmetries, we obtain the usual four independent $(a=1 \cdots 4)$ helicity amplitudes $H_{a}(\theta)$ [9].

The vector meson amplitude can be displayed as a $6 \times 4$ matrix in helicity space

$$
\mathcal{F}=\left(\begin{array}{rl|rr}
H_{21} & H_{11} & H_{3-1} & -H_{4-1} \\
H_{41} & H_{31} & -H_{1-1} & H_{2-1} \\
\hline H_{20} & H_{10} & -H_{30} & H_{40} \\
H_{40} & H_{30} & H_{10} & -H_{20} \\
\hline H_{2-1} & H_{1-1} & H_{31} & -H_{41} \\
H_{4-1} & H_{3-1} & -H_{11} & H_{21}
\end{array}\right)
$$

where the JW parity relation:

$$
\begin{gathered}
\left\langle\vec{q} \lambda_{V} \lambda_{2}|T| \vec{p} \lambda \lambda_{1}\right\rangle= \\
(-1)^{\Lambda_{f}-\Lambda_{i}}\left\langle\vec{q}-\lambda_{V}-\lambda_{2}|T| \vec{p}-\lambda-\lambda_{1}\right\rangle
\end{gathered}
$$

has been used, where $\Lambda_{i} \equiv \lambda-\lambda_{1}$ and $\Lambda_{f} \equiv \lambda_{V}-\lambda_{2}$. The rows of $\mathcal{F}$ are labeled by the final state helicities $\left(\lambda_{V}, \lambda_{2}\right)$ and the columns by the initial photon and proton helicities $\left(\lambda, \lambda_{1}\right)$. The helicity amplitudes in $\mathcal{F}$ are defined by the following,

$H_{1 \lambda_{V}} \equiv<\lambda_{V}, \lambda_{2}=+1 / 2|T| \lambda=1, \lambda_{1}=-1 / 2>$,
$H_{2 \lambda_{V}} \equiv<\lambda_{V}, \lambda_{2}=+1 / 2|T| \lambda=1, \lambda_{1}=+1 / 2>$,
$H_{3 \lambda_{V}} \equiv<\lambda_{V}, \lambda_{2}=-1 / 2|T| \lambda=1, \lambda_{1}=-1 / 2>$,
$H_{4 \lambda_{V}} \equiv<\lambda_{V}, \lambda_{2}=-1 / 2|T| \lambda=1, \lambda_{1}=+1 / 2>$,

along with the JW parity rules. The pseudoscalar meson case is described by the $\lambda_{V}=0(2 \times 4)$ part of the above matrix.

General spin observables, $\Omega$, for vector meson photoproduction can be expressed in the following trace form:

$$
\Omega=\frac{\operatorname{Tr}\left[\mathcal{F}\left(A_{\gamma} A_{N}\right) \mathcal{F}^{\dagger}\left(B_{V} B_{N^{\prime}}\right)\right]}{\operatorname{Tr}\left[\mathcal{F} \mathcal{F}^{\dagger}\right]},
$$

where the trace is over spin-space helicity quantum numbers $\lambda, \lambda_{1}, \lambda_{V}, \lambda_{2}$. This basic expression for observables is an average over a classical ensemble of particles in the beam. Interference occurs only at the quantum level for the basic two-body reaction. The matrices have the dimensions: $\mathcal{F}(6 \times 4) ; A_{\gamma} A_{N}(4 \times 4) ; \mathcal{F}^{\dagger}(4 \times 6)$; and $B_{V} B_{N^{\prime}}(6 \times 6)$.

Here $A_{\gamma}$ denotes the usual $2 \times 2$ Hermitian spin matrices $\left(1, \vec{\sigma}_{\gamma}\right)$, which describe the photon's two spin degrees of freedom. The $2 \times 2$ matrix $A_{N}$ is similarly the $\left(1, \vec{\sigma}_{N}\right)$

\footnotetext{
${ }^{1}$ We often use $\lambda_{1}= \pm 1$ to designate the nucleon's helicity, which really has the values $\lambda_{1}= \pm 1 / 2$.
} 
spin matrices, as is $B_{N^{\prime}}\left(1, \vec{\sigma}_{N^{\prime}}\right)$, which describes the recoil baryon's spin state. The vector meson matrix $B_{V}$ is a $3 \times 3$ matrix and a complete set of these is provided by the $3 \times 3$ unit operator ( $\mathbb{1}$ ), the usual spin- 1 operators $\vec{S}$, plus five independent rank- 2 operators $\tau_{2 \pm 2}, \tau_{2 \pm 1}$, and $\tau_{20}$. These operators are given in terms of the spin- 1 spherical tensor operators

$$
S_{1 \pm 1}=\mp \frac{S_{x} \pm i S_{y}}{\sqrt{2}}=\mp \frac{S_{ \pm}}{\sqrt{2}}, \quad S_{10}=S_{z},
$$

by the tensor operator coupling $\tau_{2 \mu}=\left[S_{1} \times S_{1}\right]_{2 \mu}$.

Associated with the above ensemble average, Eq. 2.6, there are density matrices describing the polarization state of each particle. For example, the incident photon and the target proton are described by:

$$
\rho_{\gamma}=\frac{I_{\gamma}}{2}\left(\mathbb{1}_{\gamma}+\vec{\sigma}_{\gamma} \cdot \vec{P}_{S}\right)
$$

and

$$
\rho_{N}=\frac{I_{N}}{2}\left(\mathbb{1}_{N}+\vec{\sigma}_{N} \cdot \vec{P}_{N}\right)
$$

The $\vec{P}_{S}$ above is the Stokes vector, which is used to describe the photon's polarization as discussed in Ref. [7]. The vector $\vec{P}_{N}$ describes the target polarization; its component in the $\hat{y}$ direction is denoted by $T \equiv \vec{P}_{N} \cdot \hat{y}$. The final state density matrices are for the final baryon:

$$
\rho_{N^{\prime}}=\frac{I_{N^{\prime}}}{2}\left(\mathbb{1}_{N^{\prime}}+\vec{\sigma}_{N^{\prime}} \cdot \vec{P}_{N^{\prime}}\right)
$$

and for the final vector meson:

$$
\rho_{V}=\frac{I_{V}}{3}\left(\mathbb{1}_{V}+\overrightarrow{\mathcal{S}} \cdot \vec{P}_{V}+3 \tau \cdot T_{V}\right) .
$$

Using the definition $\overrightarrow{\mathcal{S}} \equiv \sqrt{\frac{3}{2}} \vec{S}$, gives us a vector meson polarization

$$
\vec{P}_{V}=\frac{\operatorname{Tr}\left[\mathcal{F} \mathcal{F}^{\dagger} \overrightarrow{\mathcal{S}}\right]}{\operatorname{Tr}\left[\mathcal{F F}^{\dagger}\right]},
$$

for which each component is normalized to be $\leq 1$, since $\overrightarrow{\mathcal{S}} \cdot \overrightarrow{\mathcal{S}}=3$.

The vector meson has not only a vector polarization, but also a tensor polarization, $T_{V}^{2 \mu}$. Here $\tau \cdot T_{V} \equiv$ $\sum_{\mu}(-1)^{\mu} \tau_{2-\mu} T_{V}^{2 \mu}$, for the tensor polarization in a spherical tensor basis. Then one has

$$
T_{V}^{2 \mu}=\frac{\operatorname{Tr}\left[\mathcal{F F}^{\dagger} \tau_{2 \mu}\right]}{\operatorname{Tr}\left[\mathcal{F F}^{\dagger}\right]} .
$$

The initial and final state density matrices are simply products of the above forms,

$$
\rho_{i} \equiv \rho_{\gamma} \times \rho_{N} ; \quad \rho_{f} \equiv \rho_{V} \times \rho_{N^{\prime}},
$$


where the helicity matrix elements of the above operators are, for example, $<\lambda_{1}^{\prime}\left|\rho_{N}\right| \lambda_{1}>$, etc. Here the quantities $\vec{P}_{S}, \vec{P}_{N}, \vec{P}_{N^{\prime}}, \vec{P}_{V}$ and $T_{V}^{2 \mu}$ are real functions of angle and energy which describe how the density matrix is formed from the basic matrices (or operators) $\mathbb{1}, \vec{\sigma}_{\gamma}, \vec{\sigma}_{N}, \vec{\sigma}_{N^{\prime}}, \vec{S}_{V}$, and $\tau_{2 \mu}$, which act in their respective helicity space. These "functions" are called spin observables, with the most familiar being the polarization.

To deduce the trace form(Eq. 2.6) for the spin observables, one begins by expressing the general cross-section in terms of the above density matrices; namely,

$$
\sigma(\theta)=\varrho_{0} \frac{\operatorname{Tr} \rho_{f}}{\operatorname{Tr} \rho_{i}} ; \quad \text { where } \rho_{f}=\mathcal{F} \rho_{i} \mathcal{F}^{\dagger},
$$

and $\varrho_{0}=q /(2 k)$. Then, for a given experimental situation, one inserts the appropriate density matrices and extracts the associated spin observable in trace form, Eq. 2.6.

If one has an unpolarized beam and an unpolarized target, the initial density matrix is simply $\rho_{i}=I_{\gamma} I_{N} / 4$ and then with $\operatorname{Tr} \rho_{i}=I_{\gamma} I_{N}$ and $\rho_{f}=\frac{I_{\gamma} I_{N}}{4}\left(\mathcal{F} \mathcal{F}^{\dagger}\right)$ the differential cross section is

$$
\sigma(\theta)=\varrho_{0} \frac{1}{4} \operatorname{Tr}\left[\mathcal{F} \mathcal{F}^{\dagger}\right] \equiv \varrho_{0} \mathcal{I}(\theta) .
$$

Here we define the cross-section function $\mathcal{I}(\theta)$.

Another important case is the single spin observable called the target polarization $T$. Now one has a polarized target and the initial density matrix is $\rho_{i}=\rho_{\gamma} \times \rho_{N}=$ $\left(I_{\gamma} I_{N} / 4\right) \mathbb{1}_{\gamma}\left(\mathbb{1}_{N}+\vec{\sigma}_{N} \cdot \vec{P}_{N}\right)$. and the final density matrix is $\rho_{f}=\rho_{V} \times \rho_{N^{\prime}}=\left(I_{V} I_{N^{\prime}} / 4\right)$. Inserting these density matrices into Eq. 2.13, one finds that the target polarization is given by:

$$
\Omega^{\alpha=10, \beta=1} \rightarrow T=\frac{\operatorname{Tr}\left[\begin{array}{lll}
\mathcal{F} \mathbb{1}_{\gamma} \vec{\sigma}_{N} \cdot \hat{y} & \mathcal{F}^{\dagger}
\end{array}\right]}{\operatorname{Tr}\left[\begin{array}{l}
\mathcal{F} \mathcal{F}^{\dagger}
\end{array}\right]},
$$

where the labels $\alpha, \beta$ anticipate a subsequent discussion.

Equation 2.6 can be deduced in this same manner for all possible experimental spin setups, including not only single polarized spin cases, but also situations when two, three, or even, for the vector meson case, four spins are setup as initial states or measured in the final state. In all cases, one simply counts and measures a general cross section. Expressions for extracting the spin observables from such cross-sections are available in the literature [7,13 or simply derived from the above density matrix formulation.

\section{OBSERVABLES IN BILINEAR HELICITY PRODUCT FORM}

\section{A. The BHP form and basis matrices}

Let us now map the above trace (or ensemble average) form over to a bilinear helicity product BHP form. To 
cast the spin observables into a BHP form, one needs to insert the helicity amplitude matrix, Eq. 2.3, in the trace form expression, Eq. 2.6, with various choices of the $A, B$ spin operators. 2 Each spin observable $\Omega$ can be written in the following general BHP product form:

$$
\begin{aligned}
& \check{\Omega}^{\alpha \beta}=\Omega^{\alpha \beta} \mathcal{I}(\theta)= \pm \frac{1}{2}\left\langle H\left|\Gamma^{\alpha} \omega^{\beta}\right| H\right\rangle, \\
& = \pm \frac{1}{2} \sum_{a, b, \lambda_{V}, \lambda_{V}^{\prime}} H_{a \lambda_{V}}^{*} \Gamma_{a b}^{\alpha} \omega_{\lambda_{V} \lambda_{V}^{\prime}}^{\beta} H_{b \lambda_{V}^{\prime}} .
\end{aligned}
$$

See Eq. 2.14 for the definition of $\mathcal{I}(\theta)$. This bilinear helicity product form, which is the key expression for our analysis, often proves to be much more convenient than the original trace form of Eq. 2.6. 3 We use the symbol $\check{\Omega}$ to indicate the above profile function form, which is not to be confused with the unit vector designation as in $\hat{y}$.

For any particular spin observable, the matrices $\Gamma^{\alpha}$ are one of sixteen Hermitian $4 \times 4$ matrices and $\omega^{\beta}$ is selected from nine Hermitian $3 \times 3$ matrices. The Hermiticity of $\Gamma^{\alpha}$ and $\omega^{\beta}$ assures that Eq. 3.2 yields real observables. The $\Gamma$ matrices are Hermitian versions of the familiar sixteen Dirac matrices:

$$
\begin{gathered}
\Gamma^{1 \cdots 16}=\mathbf{1}, \gamma^{0}, i \vec{\gamma}, i \sigma^{0 x}, i \sigma^{0 y}, i \sigma^{0 z}, \sigma^{x y}, \sigma^{x z}, \sigma^{y z} \\
i \gamma^{5} \gamma^{0}, \gamma^{5} \vec{\gamma}, \gamma^{5}
\end{gathered}
$$

Note that here the four dimensional space is not that of a relativistic 4 -spinor; instead, it is the $a=1 \cdots 4$ part of the helicity amplitude $H_{a \lambda_{V}}$ "space."

The vector meson part of the helicity amplitude "space," i.e., the $\lambda_{V} \lambda_{V}^{\prime}$ part, is a three-dimensional space with nine associated Hermitian matrices $\omega^{\beta}$. These are constructed from nine spin-1 matrices as:

$$
\begin{gathered}
\omega^{\beta}=1, \sqrt{3 / 2} \vec{S}, \sqrt{6} t_{x y}, \sqrt{6} t_{x z}, \sqrt{6} t_{y z}, \\
\sqrt{3} / 2\left[ \pm\left(t_{x x}-t_{y y}\right)-\sqrt{3} t_{z z}\right],
\end{gathered}
$$

where in a Cartesian basis:

$$
t_{i j} \equiv\left(S_{i} S_{j}+S_{j} S_{i}\right) / 2-(2 / 3) \delta_{i j} .
$$

The matrices $t_{i j}$ are Cartesian versions of the earlier operators $\tau_{2 \mu}$; however, we use a different notation since $t$ acts in the helicity amplitude space (BHP) form of Eq. 3.1, while $\tau_{2 \mu}$ appears in the trace form, Eq. 2.6; see Appendix A.

\footnotetext{
${ }^{2}$ The tedious algebra was done using MAPLE.

${ }^{3}$ Note that the ket notation $\mid H>$ does not refer to a state vector, but is adopted here to represent the helicity amplitudes with the convention that $\left\langle a \lambda_{V} \mid H\right\rangle \equiv H_{a \lambda_{V}}(\theta)$.
} 
The matrices $\Gamma, \omega$ are defined to provide a complete Hermitian basis with the properties: $\operatorname{Tr}\left[\Gamma^{\alpha} \Gamma^{\beta}\right]=4 \delta_{\alpha \beta}$, and $\operatorname{Tr}\left[\omega^{\alpha} \omega^{\beta}\right]=3 \delta_{\alpha \beta}$. The last five $\omega^{5,6,7,8,9}$ matrices are the rank-2 operators associated with the vector meson's tensor polarization. The properties of the matrices $\Gamma, \omega$ are summarized in appendices.

Each value of the 16 superscripts $\alpha$ and of the 9 superscripts $\beta$ label a particular choice of spin operators $A_{\gamma}, A_{N}, B_{V}$ and $B_{N^{\prime}}$. For example, the choice $A_{\gamma}=$ $A_{N}=B_{V}=B_{N^{\prime}}=\mathbb{1}$ leads to the following BHP form

$$
\begin{aligned}
\check{\Omega}^{\alpha=1, \beta=1}=\mathcal{I}(\theta) & =\frac{1}{2} \sum_{a=1}^{4} \sum_{\lambda_{V}=0, \pm 1}\left|H_{a \lambda}\right|^{2} \\
& =\frac{1}{2}\left\langle H\left|\mathbb{1}_{N} \mathbb{1}_{V}\right| H\right\rangle,
\end{aligned}
$$

which yields the differential cross section using Eq. 2.14. The product $\check{\Omega}^{\alpha \beta} \equiv \Omega^{\alpha \beta} \mathcal{I}$, which is called a profile function, is convenient since it is proportional to bilinear products of helicity amplitudes.

Another example is $A_{\gamma}=B_{V}=B_{N^{\prime}}=\mathbb{1}$, and $A_{N}=\sigma_{y}^{N}$; then, we recover the target polarization profile function

$$
\check{\Omega}^{\alpha=10, \beta=1} \rightarrow \check{T}=T \mathcal{I}(\theta)=-\frac{1}{2}\left\langle H\left|\Gamma^{10} \omega^{1}\right| H\right\rangle,
$$

which motivated the earlier labels in Eq. 2.15.

As one ranges over all possible choices of the operators $A$ and $B$, many of the traces vanish due to parity. There are sixteen values of $\alpha$, and nine values of $\beta$. Therefore, we have $16 \times 9=144$ possible $\Gamma^{\alpha} \omega^{\beta}$ products, which correspond to 144 distinct nonzero spin observables for the $\vec{\gamma}+\vec{N} \rightarrow \vec{V}+\vec{N}^{\prime}$ reaction, at each energy and angle. For the pion case, there are only sixteen $(\alpha=1 \cdots 16)$ such observables. Since for the vector(pseudoscalar) production we only need to know 23(7) of these 144(16) to make a complete measurement, there is clearly a great redundancy in the full list of spin observables. Note that so far the only symmetry invoked has been parity. 1

\section{B. Single spin observables in BHP form}

The single spin observables for vector meson production are now given explicitly in terms of the above $\Gamma$ and $\omega$ matrices. Single spin observables involve experiments where only one of the particles is polarized. We list first the case of no particles being polarized:

\footnotetext{
${ }^{4}$ The rule for parity, based on JW conventions, can be written as $\mathcal{P}_{\gamma} \mathcal{P}_{P} \mathcal{F}=\mathcal{F}^{\dagger} \mathcal{P}_{N}$ for the pion case and $\mathcal{P}_{\gamma} \mathcal{P}_{P} \mathcal{F}=$ $\mathcal{F}^{\dagger} \mathcal{P}_{V} \mathcal{P}_{N}$ for the vector meson case, where $\mathcal{P}_{V}$ is an operator defined by its action on the spin-1 eigenstates by $\mathcal{P}_{V}\left|1 \lambda_{V}>=(-1)^{1-\lambda_{V}}\right| 1-\lambda_{V}>$.
} 


$$
\mathcal{I}=\frac{1}{2}<H\left|\Gamma^{1} \omega^{1}\right| H>
$$

which defines the cross-section and corresponds to Eq. 2.14. For convenience, we count this case as a single spin observables.

Matrices that are diagonal in the transversity amplitude basis 5 are enclosed by a box in the above equations. The significance of these boxed expressions and general properties of single spin observables will be discussed later.

The first single spin observable is the target polarization profile $\check{T} \equiv \vec{P}_{N} \cdot \hat{y} \mathcal{I}$ :

$$
\check{T}=-\frac{1}{2}<H\left|\Gamma^{10} \omega^{1}\right| H>\text {; }
$$

the next observable is the recoil polarization $\check{P}_{N^{\prime}} \equiv \vec{P}_{N^{\prime}}$. $\hat{y}^{\prime} \mathcal{I}$, e.g., the polarization of the final baryon:

$$
\check{P}_{N^{\prime}}=\frac{1}{2}<H\left|\Gamma^{12} \omega^{1}\right| H>.
$$

The next single spin observable is the polarized photon asymmetry $\check{\Sigma} \equiv \vec{\Sigma} \cdot \hat{x} \mathcal{I}$ :

$$
\check{\Sigma}=\frac{1}{2}<H\left|\Gamma^{4} \omega^{A}\right| H>.
$$

Now we turn to the final vector meson, which has a vector polarization $\check{P}_{V} \equiv \vec{P}_{V} \cdot \hat{y}^{\prime} \mathcal{I}$ :

$$
\check{P}_{V}=\frac{1}{2} \sqrt{\frac{3}{2}}<H\left|\mathbb{1} S_{y}\right| H>=\frac{1}{2}<H\left|\Gamma^{1} \omega^{3}\right| H>
$$

and also tensor polarizations, for which the transverse $(\hat{y})$ component is

$$
\begin{aligned}
\check{T}_{y y} & =\frac{1}{2}<H\left|\mathbb{1} t_{y y}\right| H> \\
& =\frac{1}{2}<H\left|\mathbb{1}\left(S_{y}^{2}-\frac{2}{3}\right)\right| H> \\
& =\frac{1}{2}<H\left|\Gamma^{1}\left[\frac{3 \omega^{A}-\omega^{1}}{6}\right]\right| H>,
\end{aligned}
$$

with the spherical tensor components given by:

$$
\check{T}_{21}=\sqrt{\frac{1}{6}}<H\left|\Gamma^{1} \omega^{6}\right| H>,
$$

\footnotetext{
${ }^{5}$ Transversity amplitudes are similar to helicity amplitudes except that the axis of spin quantization is the transverse rather than the particle's momentum direction. Hence, transversity amplitudes are defined using the axis of quantization perpendicular to the scattering plane $(\hat{y})$ instead of using the particle momentum directions $\left(\hat{z}, \hat{z}^{\prime}\right)$.
} 
and

$$
\begin{aligned}
\check{T}_{22}= & \frac{1}{\sqrt{3}} \frac{1}{4}<H\left|\Gamma^{1}\left[\omega^{8}-\omega^{9}\right]\right| H>, \\
\check{T}_{20} & =-\frac{1}{\sqrt{6}}<H\left|\Gamma^{1}\left[\omega^{8}+\omega^{9}\right]\right| H>, \\
& =\frac{3}{2}<H\left|t_{z z}\right| H>, \\
& =\frac{3}{2}<H\left|S_{z}^{2}-\frac{2}{3}\right| H>.
\end{aligned}
$$

Only eight of the above single spin observables are independent, since we have $\check{T}_{2 \mu}=(-1)^{\mu} \check{T}_{2-\mu}$.

The following characteristic combinations of the $(3 \times 3)$ $\omega^{1,8,9}$ matrices are used above and later:

$$
\left(\begin{array}{l}
\omega^{A} \\
\omega^{B} \\
\omega^{C}
\end{array}\right) \equiv \frac{1}{3}\left(\begin{array}{ccc}
1 & (1-\sqrt{3}) & (1+\sqrt{3}) \\
(1-\sqrt{3}) & (1+\sqrt{3}) & 1 \\
(1+\sqrt{3}) & 1 & (1-\sqrt{3})
\end{array}\right)\left(\begin{array}{c}
\omega^{1} \\
\omega^{8} \\
\omega^{9}
\end{array}\right),
$$

which is a unitary transformation in the $\omega$ basis.

\section{Double spin observables in BHP form}

The double spin observables are now presented using the notation:

$$
\check{C}_{i j}^{\gamma N} \equiv \frac{1}{2}<H\left|\mathcal{C}_{i j}^{\gamma N}\right| H>
$$

The $\check{C}$ is used to designate the spin observable profile function, while the symbol $\mathcal{C}$ is used to designate a matrix in the helicity $H_{a \lambda_{V}}$ space. In addition, superscripts are used to stipulate the two polarized particles in the reaction. Finally, a matrix display will be used to present the Cartesian components $(i, j)$ of the various spin observables; for example, instead of writing $\mathcal{C}_{i j}$ as $\mathcal{C}_{x x}, \mathcal{C}_{x y}, \mathcal{C}_{x z}$, these are presented below as the top row of a matrix. These conventions allow us to present the 99 double spin observables in a relatively compact form. .

\section{Beam-target observables}

Let us start with the beam-target $(\gamma-N)$ double spin observables. The Cartesian terms are:

\footnotetext{
${ }^{6}$ Of the 99 possible double spin observables for vector meson photoproduction, 51 are nonzero, all others vanish by virtue of parity considerations. The nonzero 51 observables are not in general independent of the other spin observables.
} 


$$
\mathcal{C}^{\gamma N}=\left(\begin{array}{ccc}
0 & -\Gamma^{12} \omega^{A} & 0 \\
\Gamma^{5} \omega^{A} & 0 & \Gamma^{3} \omega^{A} \\
\Gamma^{11} & 0 & \Gamma^{9} \\
\omega^{1} & 0
\end{array}\right),
$$

Note that there are 9 entries in the above Cartesian component $(x, y, z)$ display, but four vanish; namely, $\mathcal{C}_{x x}=$ $\mathcal{C}_{x z}=\mathcal{C}_{y y}=\mathcal{C}_{z y}=0$. Also note that a set of five independent $\Gamma^{\alpha}$ matrices appear above for $\alpha=3,5,9,11,12$, along with $\omega^{1}$ and the linear combination $\omega^{A}$ matrix (Eq. 3.17). Here both particles are in the initial state and the Cartesian components refer to the initial $(x, y, z)$ axes of Fig. 1. Of the nine possible beam-target spin observables, only five are nonzero. Near the vector meson production threshold, only one of the above double spin observables is nonzero; namely, $\mathcal{C}_{z z}^{\gamma N} \equiv \Gamma^{9} \omega^{1}$, see Ref. [16].

\section{Beam-recoil observables}

For the beam-recoil $\left(\gamma-N^{\prime}\right)$ double spin observables, we have a similar display, $\check{C}_{i j}^{\gamma N^{\prime}} \equiv \frac{1}{2}<H\left|\mathcal{C}_{i j}^{\gamma N^{\prime}}\right| H>$

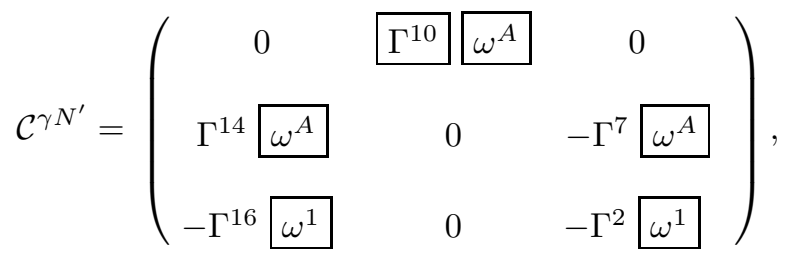

but a different set of $\Gamma$ matrices appear; namely, $\alpha=$ $2,7,10,14,16$. The $\omega^{1}$ and $\omega^{A}$ matrices appear again as in Eq. 3.17. Here the Cartesian components of the initial photon refers to the $(x, y, z)$ axes of Fig. 1, while the final axes $\left(x^{\prime}, y^{\prime}, z^{\prime}\right)$ are used for the recoil baryon $N^{\prime}$. Therefore, we can extract a particular double spin observable from Eq. 3.18 as:

$$
\check{C}_{x y^{\prime}}^{\gamma N^{\prime}}=\frac{1}{2}<H\left|\Gamma^{10} \omega^{A}\right| H>\equiv \check{\Omega}^{10, A},
$$

where

$$
\check{\Omega}^{10, A}=\frac{1}{3}\left[\check{\Omega}^{10,1}+(1-\sqrt{3}) \check{\Omega}^{10,8}+(1+\sqrt{3}) \check{\Omega}^{10,9}\right] .
$$

Of the nine possible beam-recoil spin observables, only five are nonzero. Near the vector meson production threshold, only two of the above double spin observables are nonzero; namely, $\mathcal{C}_{z z^{\prime}}^{\gamma N^{\prime}} \equiv-\Gamma^{2} \omega^{1}$, and $\mathcal{C}_{z x^{\prime}}^{\gamma N^{\prime}} \equiv$ $-\Gamma^{16} \omega^{1}$, see Ref. [16].

\section{Target-recoil observables}

For the target-recoil $\left(N-N^{\prime}\right)$ double spin observables, we have yet another set of four $\Gamma$ matrices appearing: $\check{C}_{i j}^{N N^{\prime}} \equiv \frac{1}{2}<H\left|\mathcal{C}_{i j}^{N N^{\prime}}\right| H>$ with 


$$
\mathcal{C}^{N N^{\prime}}=\left(\begin{array}{ccc}
-\Gamma^{6} & 0 & -\Gamma^{13} \\
0 & -\Gamma^{4} & 0 \\
\Gamma^{8} & 0 & \Gamma^{15}
\end{array}\right) \omega^{1} .
$$

This time we have $\Gamma^{\alpha}$ 's with $\alpha=4,6,8,13,15$. Here the Cartesian components of the initial nucleon $N$ refers to the $(x, y, z)$ axes of Fig. 1, while the final axes $\left(x^{\prime}, y^{\prime}, z^{\prime}\right)$ are used for the recoil baryon $N^{\prime}$. A particular double spin observable from Eq. 3.19 is:

$$
\check{C}_{y y^{\prime}}^{N N^{\prime}}=-\frac{1}{2}<H\left|\Gamma^{4} \omega^{1}\right| H>\equiv \check{\Omega}^{4,1} .
$$

This is often called $D_{n n}$, where the subscript $n$ refers to the normal to the scattering plane, which in our case is $\hat{y}=\hat{y}^{\prime}$. Of the nine possible target-recoil spin observables, only five are nonzero. Near the vector meson production threshold, all five of the above double spin observables are nonzero, see Ref. 16.

\section{Beam-vector meson observables}

We now consider the first double spin observable which involves both a polarized photon and a final polarized vector meson. We again display the Cartesian components as a $i, j$ array, with the spin profile function $\check{C}_{i j}$ constructed from the helicity space matrix $\mathcal{C}_{i j}$ as $\check{C}_{i j}^{\gamma V} \equiv \frac{1}{2}<H\left|\mathcal{C}_{i j}^{\gamma V}\right| H>$. However, since the final vector meson has not only a vector polarization, but also five possible tensor polarization components the matrix now involves the following $3 \times 8$ matrix. The three rows refer to the three Cartesian components $i=1,2,3$ or $x, y, z$ of the photon's Stokes vector, while the first three columns $j=1,2,3$ refer to the vector meson's three polarization components $\vec{P}_{V}$. The last five columns $j=4,5,6,7,8$ refer to the tensor polarization of the vector meson $\sqrt{6} T_{x y}^{V}, \sqrt{6} T_{x z}^{V}, \sqrt{6} T_{y z}^{V}, \frac{\sqrt{3}}{2}\left( \pm\left(T_{x x}^{V}+T_{y y}^{V}\right)-\sqrt{3} T_{z z}^{V}\right.$

The Cartesian components for the $\gamma$ refer to the original $(x, y, z)$ axes and for the final vector particle refer to the final $\left(x^{\prime}, y^{\prime}, z^{\prime}\right)$ axes of Fig. 1. The result is:

$$
\mathcal{C}^{\gamma V}=\left(\begin{array}{lll}
\Gamma^{4} & \Gamma^{4} \Gamma^{1}
\end{array}\right)\left(\begin{array}{cccccccc}
0 & \omega^{3} & 0 & 0 & \omega^{6} & 0 & \omega^{B} & \omega^{C} \\
-\omega^{7} & 0 & \omega^{5} & \omega^{4} & 0 & \omega^{2} & 0 & 0 \\
\omega^{2} & 0 & \omega^{4} & \omega^{5} & 0 & \omega^{7} & 0 & 0
\end{array}\right) .
$$

The spin observable in the photon density matrix of Eq. 2.7 has the three Cartesian components; whereas, the vector meson density matrix Eq. 2.10 has three vector plus five tensor polarization spin observables. Again, that is the origin of the $3 \times 8$ nature of the above double spin observable display.

For convenience, we separate the helicity space $H_{a \lambda_{V}}$ into separate $<\lambda_{V}|\omega| \lambda_{V}^{\prime}>$ and $\Gamma_{a b}$ matrices. To illustrate our notation, the following double spin observable

$$
\check{C}_{x y^{\prime}}^{\gamma V}=\frac{1}{2}<H\left|\Gamma^{4} \omega^{3}\right| H>=\check{\Omega}^{4,3}
$$


can be extracted from Eq. 3.20. Another example is

$$
\check{C}_{z 4}^{\gamma V}=\frac{1}{2}<H\left|\Gamma^{1} \omega^{5}\right| H>=\check{\Omega}^{1,5}
$$

Of the 24 possible beam-vector meson spin observables, only 12 are nonzero. Near the vector meson production threshold, seven of the above twelve double spin observables are nonzero; namely, $\check{C}_{z, x^{\prime}}^{\gamma V}, \check{C}_{z, z^{\prime}}^{\gamma V}, \check{C}_{y, 4}^{\gamma V}, \check{C}_{x, 5}^{\gamma V}, \check{C}_{y, 6}^{\gamma V}, \check{C}_{x, 7}^{\gamma V}, \check{C}_{x, 8}^{\gamma V}$, see Ref. 16.

\section{Recoil-vector meson observables}

Similarly, the recoil-vector meson $\left(N^{\prime}-V\right)$ case involves a final polarized baryon $\left(N^{\prime}\right)$ and the $3+5$ vector plus tensor components of the final vector meson. In this case the Cartesian components for both the $N^{\prime}$ and the final vector particle refer to the final $\left(x,{ }^{\prime} y^{\prime}, z^{\prime}\right)$ axes of Fig. 1. Thus, a $3 \times 8$ display appears again, where the double polarization spin observable $\check{C}$ is expressed in terms of a helicity amplitude space matrix $\mathcal{C}_{i j}$ : $\check{C}_{i j}^{N^{\prime} V} \equiv \frac{1}{2}<H\left|\mathcal{C}_{i j}^{N^{\prime} V}\right| H>$ with

$\mathcal{C}^{N^{\prime} V}=-\left(\begin{array}{ll}\Gamma^{16} & -\Gamma^{12} \\ \Gamma^{2}\end{array}\right)\left(\begin{array}{cccccccc}\omega^{2} & 0 & \omega^{4} & \omega^{5} & 0 & \omega^{7} & 0 & 0 \\ 0 & \omega^{3} & 0 & 0 & \omega^{6} & 0 & \omega^{8} & \omega^{9} \\ \omega^{2} & 0 & \omega^{4} & \omega^{5} & 0 & \omega^{7} & 0 & 0\end{array}\right)$

To illustrate our notation again, the following double spin observable

$$
\check{C}_{x^{\prime}, z^{\prime}}^{N^{\prime} V}=-\frac{1}{2}<H\left|\Gamma^{16} \omega^{4}\right| H>\equiv \check{\Omega}^{16,4}
$$

can be extracted from Eq. 3.20. Another example is

$$
\check{C}_{z^{\prime}, 4}^{N^{\prime} V}=-\frac{1}{2}<H\left|\Gamma^{2} \omega^{5}\right| H>\equiv \check{\Omega}^{2,5}
$$

Of the 24 possible recoil-vector meson spin observables, only 12 are nonzero. Near the vector meson production threshold, all of the above twelve double spin observables are nonzero, Ref. [16].

\section{Target-vector meson observables}

Finally, the target-vector meson $(N-V)$ case has the same type of display with the double spin observable related to a helicity amplitude space matrix by

$$
\check{C}_{i j}^{N V} \equiv \frac{1}{2}<H\left|\mathcal{C}_{i j}^{N V}\right| H>
$$

with again a $3 \times 8$ matrix

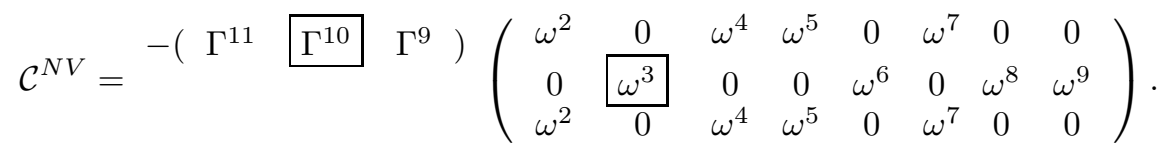


Of the 24 possible target-vector meson spin observables, only 12 are nonzero. Near the vector meson production threshold, ten of the above twelve double spin observables are nonzero; namely, $\check{C}_{x x^{\prime}}^{N V}, \check{C}_{z x^{\prime}}^{N V}, \check{C}_{y y^{\prime}}^{N V}, \check{C}_{x z^{\prime}}^{N V}, \check{C}_{z z^{\prime}}^{N V}, C_{y, z z}^{N V}, C_{x, y z}^{N V}, C_{y, x z}^{N V}, C_{y, x x}^{N V}, C_{x, x y}^{N V}$ see Ref. [16].

The significance of the boxed matrices will become clear when we discuss the transversity amplitudes.

\section{Some general remarks}

There are 99 possible double spin observables, which reduce to 51 after use of parity conservation. The total number of nonzero observables near threshold is 37 out of these 51, see Ref. [16]. There are 18 single spin observables (we count the cross-section as a single spin observable), which reduce to 8 using parity. Of these 8 , five are nonzero near or at threshold, which include the cross-section plus 4 vector meson spin observables; namely, $T_{x x}, T_{y y}, T_{z z}$, and $T_{x z}$. Since there are 12 complex amplitudes, one needs $24-1=23$ independent measurements to determine the photoproduction amplitudes completely, with one overall arbitrary phase. At first glance, it would seem that with 8 single and 51 double spin observables, it suffices to do just selected single and double spin measurements to completely determine the 12 amplitudes. This proves not to be the case.

The reason for this conclusion is that many of these spin observables yield redundant information. In Section $\mathrm{V}$, it is shown that spin observables of the same "phase class" can yield redundant information and that one needs to go beyond single plus double spin observables to obtain a complete set of experiments. The "phase class" will be defined later.

Triple and also quadruple spin observables have been derived and complete the full set of $16 \times 9=144$ spin observables. The results are given in Appendix F. Before use of parity there are 243 triple spin observables; after use of parity conservation there are 123. Before use of parity there are 216 quadruple spin observables; after use of parity conservation there are 108. Many of these double, triple and quadruple spin observables involve linear combinations or the same BHP forms $\Gamma^{\alpha} \times \omega^{\beta}$ that appear in other single and double spin observables. That reveals many relations between spin observables and is an important advantage of the BHP display.

The basic question is: which of these $8+51+123+$ $108=290$ observables can and need to be measured to determine the 12 complex amplitudes? Clearly, 23 measurements are needed at each energy and angle, but they have to be selected to yield independent information. This problem is dealt with in Section V. In addition, one can ask which observables are expected to have nodes not only at the $0^{\circ}$ and $180^{\circ}$ endpoints, but also in between? That and related questions are addressed next. 


\section{NODAL STRUCTURE-LEGENDRE CLASS}

Having expressed the spin observables for vector meson photoproduction in BHP form using the basic matrices $\Gamma$ and $\omega$, we can analyze these matrices for insights as to the "nodal structure" of observables. Note that the original $\Gamma$ and $\omega$ matrices can be organized into groups according to their common "shape;" let us call these groupings "Legendre classes." For example, the following matrices are of diagonal $(D)$ shape: $\Gamma^{1} \Gamma^{2} \Gamma^{9} \Gamma^{15}$; whereas, the following are antidiagonal $(A D): \Gamma^{3} \Gamma^{4} \Gamma^{6} \Gamma^{7}$. The remaining eight matrices are either of one class (called PL for left parallelogram form): $\Gamma^{10} \Gamma^{11} \Gamma^{13} \Gamma^{14}$, or of another class (called PR for right parallelogram shape): $\Gamma^{5}$ $\Gamma^{8} \Gamma^{12} \Gamma^{16}$. Similarly for the $\omega$ space, one has matrices of diagonal $(D)$ shape: $\omega^{1} \omega^{4}$, antidiagonal $(A D)$ shape: $\omega^{5} \omega^{A}$, crossed shape $(X): \omega^{8} \omega^{9}, \omega^{B} \omega^{C}$, and diamond or polygon $(P)$ shape: $\omega^{2} \omega^{3}, \omega^{6} \omega^{7}$. Explicit $\Gamma$ and $\omega$ matrices are presented in Appendix B, where they are grouped together by their common, $(D, A D, P L, P R)$-for $\Gamma$ or $(D, A D, X, P)$-for $\omega$, shapes.

We stress classification of the matrices by their shape because classes of observables involving $\Gamma$ and $\omega$ matrices of the same shape have angular dependencies given by related mixtures of the same Wigner $d$-functions. To illustrate this remark, let us consider the general spin observable $\check{\Omega}^{\alpha, \beta}$, which is given in terms of the helicity amplitudes $H_{a, \lambda_{V}}(\theta)$ by Eq. 3.2. The helicity amplitudes have the following partial wave expansion

$$
H_{a \lambda_{V}}(\theta)=\sum_{J_{1}}\left(2 J_{1}+1\right) H_{a \lambda_{V}}^{J_{1}} d_{\Lambda_{a f}, \Lambda_{a i}}^{J_{1}}(\theta),
$$

where $\Lambda_{a f}$ and $\Lambda_{a i}$ take on the following values:

$$
\Lambda_{a f}=\lambda_{V}-\zeta_{a} \text { and } \Lambda_{a i}=\xi_{a}
$$

where $\zeta_{1}=\zeta_{2}=-\zeta_{3}=-\zeta_{4}=1 / 2$ and $\xi_{2}=\xi_{4}=$ $1 / 2, \xi_{1}=\xi_{3}=3 / 2$, see Eq. 2.5. Using the above expansion for $H_{a \lambda_{V}}$ and $H_{b \lambda_{V}^{\prime}}$ in Eqs. 3.1, 3.2, we have:

$$
\begin{aligned}
\check{\Omega}^{\alpha \beta}(\theta)= & \pm \frac{1}{2} \sum_{a, b, \lambda_{V}, \lambda_{V}^{\prime}} \sum_{J_{1}, J_{2}} H_{a \lambda_{V}}^{J_{1 *}} H_{b \lambda_{V}^{\prime}}^{J_{2}} \Gamma_{a b}^{\alpha} \omega_{\lambda_{V} \lambda_{V}^{\prime}}^{\beta} \\
& d_{\lambda_{V}-\zeta_{a}, \xi_{a}}^{J_{1}}(\theta) d_{\lambda_{V}^{\prime}-\zeta_{b}, \xi_{b}}^{J_{2}}(\theta) .
\end{aligned}
$$

This bilinear form can be combined to extract the dependence on a single Wigner- $d$. One finds:

$$
\begin{aligned}
\check{\Omega}^{\alpha \beta}(\theta)= & \sum_{\mathcal{J}, \Lambda, \Lambda^{\prime}} \sum_{a, b, \lambda_{V}, \lambda_{V}^{\prime}}(2 \mathcal{J}+1) \\
& d_{\Lambda, \Lambda^{\prime}}^{\mathcal{J}}(\theta) \times \Xi_{\Lambda, \Lambda^{\prime}}^{\mathcal{J}, \alpha \beta}\left[a b ; \lambda_{V}, \lambda_{V}^{\prime}\right],
\end{aligned}
$$

with

$$
\begin{array}{cc}
\Xi_{\Lambda, \Lambda^{\prime}}^{\mathcal{J}, \alpha \beta}\left[a b ; \lambda_{V}, \lambda_{V}^{\prime}\right] & \equiv \\
\pm \frac{1}{2} \sum_{J_{1}, J_{2}} \Gamma_{a b}^{\alpha} \omega_{\lambda_{V} \lambda_{V}^{\prime}}^{\beta} & H_{a \lambda_{V}}^{J_{1 *}} H_{b \lambda_{V}^{\prime}}^{J_{2}} \\
\left(\begin{array}{ccc}
J_{1} & J_{2} & \mathcal{J} \\
\lambda_{V}-\zeta_{a} & \lambda_{V}^{\prime}-\zeta_{b} & \Lambda
\end{array}\right) & \left(\begin{array}{ccc}
J_{1} & J_{2} & \mathcal{J} \\
\xi_{a} & \xi_{b} & \Lambda^{\prime}
\end{array}\right) .
\end{array}
$$


Note that $\Lambda$ depends on the helicity labels $a, b$ and $\lambda_{V}, \lambda_{V}^{\prime}$, while $\Lambda^{\prime}$ depends only on the helicity labels $a, b$.

To understand the consequence of the above result, select a particular choice of spin observable by designating the associated values of $\alpha$, and $\beta$; for example, take 10, 1 for the target polarization, see Eq. 2.15. Now consider the full family of $\Gamma \times \omega$ matrices with the same matrix "shape" for both $\Gamma$ and $\omega$. That family is called a "Legendre class." For the target polarization case, the matrix products $\Gamma^{10,11,13,14} \times \omega^{1,4}$ are all of the same shape and thus the associated spin observables form a "Legendre class." (The members of this target polarization class will be discussed later.)

Since all members of a "Legendre class" vanish for the same $\left(a, b ; \lambda_{V} \lambda_{V}^{\prime}\right)$ values, they are all formed from the same set of Wigner $d$-functions, $d_{\Lambda, \Lambda^{\prime}}^{\mathcal{J}}(\theta)$ of Eqs. 4.3.4.4. If, for example, every member of that set of Wigner $d$ functions vanishes at $0^{\circ}$ and $180^{\circ}$, then every member of that "Legendre class" of spin observables will also vanish at $0^{\circ}$ and $180^{\circ}$. Similarly, if every member of that set of Wigner $d$-functions has a zero or a node at $90^{\circ}$, then every member of that "Legendre class" of spin observables will also have a zero or a node at $90^{\circ}$. These observations follow from the fact that these families of spin observables are all expressed by various combinations (see $\Xi$, above) of the same set of Wigner $d$-functions. The mixture coefficients, $\Xi$, do depend on the partial wave helicity amplitudes, which is how dynamics of the reaction affects the detailed angular dependencies. If one truncates the partial wave expansion due to either threshold or resonance considerations, then the blend of Wigner $d$ 's is strongly restricted and one can demonstrate explicit associated angle dependencies of the spin observable profiles. For example, if only one $J_{1}, J_{2}$ set of partial wave helicity amplitudes are nonzero, then using the triangle rule $\mathcal{J}=J_{1}+J_{2} \cdots J_{1}-J_{2}$, only a limited number of $\mathcal{J}$ values appear, which severely restricts the nodal structure. That allows one to test and extract specific dynamical information from the nodal structure and energy evolution of spin observables (see Ref. [8]).

Thus the angular dependencies of spin observables can be grouped into classes [7, 8 with the same potential nodal structure (hence the nomenclature Legendre class). To examine the general role of resonances on the nodal structure of spin observables, it is most convenient to introduce explicit orbital angular momentum quantum numbers. Therefore, in a separate paper Ref. [16] the electric and magnetic multipole amplitudes for vector meson photoproduction are studied in detail.

Another way of examining the angular dependence of a Legendre class of spin observables is to return to the partial wave expansion, Eq. 4.1. For a given Legendre class, only selected values of $a$ and $\lambda_{V}$ appear. Thus, one can pick the corresponding helicity amplitudes from the first column of Table II, then proceed to the partial wave helicity amplitudes and the associated Wigner $d-$ functions in the second and third columns. The fourth 
column gives the range of $J$ needed for that amplitude to contribute; while the last two columns indicate the value of the Wigner $d$-function at the $0^{\circ}$ and $180^{\circ}$ endpoints.

TABLE I. Partial Wave expansion of the helicity amplitudes. The associated Wigner $d$-functions are shown along with an indication of their values at the $0^{\circ}$ and $180^{\circ}$ endpoints. Six of the helicity amplitudes $H_{a, \lambda_{V}}(\theta)$ vanish at both endpoints; namely, $H_{1,1}, H_{1,0}, H_{2,-1}, H_{3,0}, H_{3,-1}, H_{4,1}$. Three of the helicity amplitudes vanish only at the $0^{\circ}$ endpoint; namely, $H_{1,-1}, H_{2,0}, H_{4,-1}$. Three of the helicity amplitudes vanish only at the $180^{\circ}$ endpoint; namely, $H_{2,1}, H_{3,1}, H_{4,0}$.

\begin{tabular}{lccccc}
\hline \hline$H_{a, \lambda_{V}}(\theta)$ & $H_{a, \lambda_{V}}^{J}$ & $d_{\Lambda_{f}, \Lambda_{i}}^{J}(\theta)$ & $J$ & $0^{\circ}$ & $180^{\circ}$ \\
\hline$H_{1,1}(\theta)$ & $H_{1,1}^{J}$ & $d_{1 / 2,3 / 2}^{J}(\theta)$ & $J \geq 3 / 2$ & 0 & 0 \\
$H_{2,1}(\theta)$ & $H_{2,1}^{J}$ & $d_{1 / 2,1 / 2}^{J}(\theta)$ & $J \geq 1 / 2$ & 1 & 0 \\
$H_{3,1}(\theta)$ & $H_{3,1}^{J}$ & $d_{3 / 2,3 / 2}^{J}(\theta)$ & $J \geq 3 / 2$ & 1 & 0 \\
$H_{4,1}(\theta)$ & $H_{4,1}^{J}$ & $d_{3 / 2,1 / 2}^{J}(\theta)$ & $J \geq 3 / 2$ & 0 & 0 \\
\hline$H_{1,0}(\theta)$ & $H_{1,0}^{J}$ & $d_{-1 / 2,3 / 2}^{J}(\theta)$ & $J \geq 3 / 2$ & 0 & 0 \\
$H_{2,0}(\theta)$ & $H_{2,0}^{J}$ & $d_{-1 / 2,1 / 2}^{J}(\theta)$ & $J \geq 1 / 2$ & 0 & 1 \\
$H_{3,0}(\theta)$ & $H_{3,0}^{J}$ & $d_{1 / 2,3 / 2}^{J}(\theta)$ & $J \geq 3 / 2$ & 0 & 0 \\
$H_{4,0}(\theta)$ & $H_{4,0}^{J}$ & $d_{1 / 2,1 / 2}^{J}(\theta)$ & $J \geq 1 / 2$ & 1 & 0 \\
\hline$H_{1,-1}(\theta)$ & $H_{1,-1}^{J}$ & $d_{-3 / 2,3 / 2}^{J}(\theta)$ & $J \geq 3 / 2$ & 0 & 1 \\
$H_{2,-1}(\theta)$ & $H_{2,-1}^{J}$ & $d_{-3 / 2,1 / 2}^{J}(\theta)$ & $J \geq 3 / 2$ & 0 & 0 \\
$H_{3,-1}(\theta)$ & $H_{3,-1}^{J}$ & $d_{-1 / 2,3 / 2}^{J}(\theta)$ & $J \geq 3 / 2$ & 0 & 0 \\
\hline \hline & $H_{4,-1}^{J}(\theta)$ & $d_{-1 / 2,1 / 2}^{J}(\theta)$ & $J \geq 1 / 2$ & 0 & 1 \\
\hline & & & & \\
\hline
\end{tabular}


Using this table, one can deduce which observables are zero at the endpoints. For example, consider the Legendre class of observables for which both $\Gamma^{\alpha}$ and $\omega^{\beta}$ are diagonal, e.g., the class: $\Gamma^{1,2,9,15} \times \omega^{1,4}$. These observables depend on linear combinations of $\sum_{a \lambda_{V}} \pm\left|H_{a, \lambda_{V}}\right|^{2}$. From Table if it follows that the associated observables do not necessarily vanish at the end points. In this diagonal Legendre class the single and double observables are: $\mathcal{I}, \mathcal{C}_{z z^{\prime}}^{\gamma V}, \mathcal{C}_{z z^{\prime}}^{\gamma N^{\prime}}, \mathcal{C}_{z^{\prime} z^{\prime}}^{N^{\prime} V}, \mathcal{C}_{z z}^{\gamma N}, \mathcal{C}_{z z^{\prime}}^{N V}$ and $\mathcal{C}_{z z^{\prime}}^{N N^{\prime}}$. This information is extracted from Tables III [II], where the relation between the $\alpha$ and $\beta$ choices of the matrices are related to explicit spin observables.

Another example of how to use Tables II III to deduce the endpoint behavior of a given Legendre class of observables is seen by examining the Legendre class of observables for which both $\Gamma^{\alpha}$ and $\omega^{\beta}$ are antidiagonal, e.g., the class: $\Gamma^{3,4,6,7} \times \omega^{5, A}$. Only the helicity amplitude products with $a, b=(1,4),(2,3)$ and $\lambda_{V}^{\prime}=-\lambda_{V}$ appear for these observables. Now using Table those products, it follows that this Legendre class involved bilinear helicity products that vanish at both $0^{\circ}$ and $180^{\circ}$. From Tables IIIIII, we learn that the single, double and triple spin observables of this class are: $\Sigma, \mathcal{C}_{y z^{\prime}}^{\gamma V}, \mathcal{C}_{y z^{\prime}}^{\gamma N^{\prime}}, \mathcal{C}_{y z}^{\gamma N}, \mathcal{C}_{x y^{\prime} 4}^{N N^{\prime} V}, \mathcal{C}_{x z z^{\prime}}^{N N^{\prime} V}, \mathcal{C}_{y x^{\prime} 4}^{N N^{\prime} V}, \mathcal{C}_{x z^{\prime} z^{\prime}}^{\gamma N^{\prime} V} \quad$ and $\mathcal{C}_{x z z^{\prime}}^{\gamma N N^{\prime}}$.

The procedure consists of using Tables III along with the Legendre class information to determine the endpoint rules. Alternately, one can use the general results Eqs. 4.3 4.4.

\section{TRANSVERSITY, PHASE CLASS AND COMPLETE EXPERIMENTS}

\section{A. Transversity}

\section{Pseudoscalar meson transversity}

Let us deal with the question of which experiments are needed to determine the magnitude and phase of the 12 helicity amplitudes for vector meson production. Clearly, 23 experiments are needed at each energy and angle. It is well known that for pseudoscalar meson photoproduction, it is possible to determine the magnitude of the four transversity amplitudes by measuring all four single spin observables. The remaining three phases (one overall phase is arbitrary) can be determined by selecting three additional double spin observables, following the BDS 10,11] rules. The pseudoscalar meson case can be recovered from Eqs. 3.7 3.10, by setting $\omega^{A} \rightarrow 1$ and omitting all observables which involve a final meson spin of 1 . In that limit, the BDS rules can be understood by performing a unitary transformation on the $\Gamma$ matrices along with a unitary transformation on the helicity amplitudes to generate the transversity amplitudes $|\widetilde{H}\rangle$ : 


$$
\left|\widetilde{H}_{a}>\equiv \sum_{b} \mathbf{U}_{a, b}^{(4)}\right| H_{b}>,
$$

and a new set of $\Gamma$ matrices

$$
\widetilde{\Gamma}^{\alpha} \equiv \mathbf{U}^{(4)} \Gamma^{\alpha} \mathbf{U}^{\dagger(4)} .
$$

The sixteen spin observables are invariant under such a unitary transformation in "helicity space:"

$$
\check{\Omega}^{\alpha} \propto<H\left|\Gamma^{\alpha}\right| H>\equiv<\widetilde{H}\left|\widetilde{\Gamma}^{\alpha}\right| \widetilde{H}>.
$$

The physically meaningful unitary operator is the transversity choice,

$$
\mathbf{U}^{(4)}=\frac{1}{2}\left(\begin{array}{rrrr}
1 & -i & i & 1 \\
1 & i & -i & 1 \\
1 & i & i & -1 \\
1 & -i & -i & -1
\end{array}\right),
$$

which involves rotating the helicity quantization axis $(\hat{z}$ and $\hat{z}^{\prime}$ ) to the direction normal to the scattering plane $\hat{y}=\hat{y}^{\prime}$, see Fig. 1 . With the above $4 \times 4$ unitary transversity transformation, the following matrices now are diagonal:

$$
\widetilde{\Gamma}^{1} \widetilde{\Gamma}^{4} \widetilde{\Gamma}^{10} \quad \widetilde{\Gamma}^{12} ;
$$

whereas, the following are now antidiagonal:

$$
\begin{array}{llll}
\widetilde{\Gamma}^{2} & \widetilde{\Gamma}^{7} & \widetilde{\Gamma}^{14} & \widetilde{\Gamma}^{16} .
\end{array}
$$

The remaining eight matrices are either of one class (called PL for left parallelogram form):

$$
\begin{array}{llll}
\widetilde{\Gamma}^{6} & \widetilde{\Gamma}^{8} & \widetilde{\Gamma}^{13} & \widetilde{\Gamma}^{15},
\end{array}
$$

or of another class (called PR for right parallelogram form):

$$
\widetilde{\Gamma}^{3} \widetilde{\Gamma}^{5} \widetilde{\Gamma}^{9} \widetilde{\Gamma}^{11}
$$

\section{Vector meson transversity}

The above procedure can now be extended to the vector meson case by introducing an additional unitary transversity transformation in the $3 \times 3$ space

$$
\mathbf{U}^{(3)}=\frac{1}{2}\left(\begin{array}{ccc}
-1 & \sqrt{2} i & 1 \\
-\sqrt{2} i & 0 & -\sqrt{2} i \\
1 & \sqrt{2} i & -1
\end{array}\right),
$$

which makes the $\hat{y}$-axis the quantization axis for the spin1 meson. Correspondingly, there is now a transformation in "helicity space:" $<H\left|\Gamma^{\alpha} \omega^{\beta}\right| H>\equiv<\widetilde{H}\left|\widetilde{\Gamma}^{\alpha} \widetilde{\omega}^{\beta}\right| \widetilde{H}>$, with

$$
\left|\widetilde{H}_{a, \lambda_{V}}>\equiv \sum_{b, \lambda_{V}^{\prime}} \mathbf{U}_{a, b}^{(4)} \mathbf{U}_{\lambda_{V}, \lambda_{V}^{\prime}}^{(3)}\right| H_{b, \lambda_{V}^{\prime}}>
$$


For the vector meson part of the transversity $\widetilde{\omega}$ space, the following matrices now have diagonal $(D)$ form: $\widetilde{\omega}^{1} \widetilde{\omega}^{3}$ $\widetilde{\omega}^{A}$; antidiagonal $(A D)$ form: $\widetilde{\omega}^{6}$; crossed $(X)$ form:

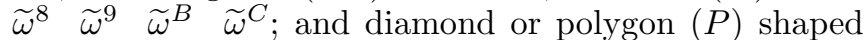
form: $\widetilde{\omega}^{2} \quad \widetilde{\omega}^{4} \quad \widetilde{\omega}^{5} \quad \widetilde{\omega}^{7}$. We have extended the definition of transversity amplitudes to the case of a vector meson. The original expressions for the spin observable profiles $\check{\Omega}^{\alpha \beta}$ are of the same form as given in Eqs. 3.7 3.22, except that the helicity amplitudes $H_{a \lambda_{V}}$ are replaced by the transversity amplitudes $\widetilde{H}_{a, \lambda_{V}}$, and the matrices are replaced by $\Gamma^{\alpha} \rightarrow \widetilde{\Gamma}^{\alpha}$ and $\omega^{\beta} \rightarrow \widetilde{\omega}^{\beta}$. In this new representation the diagonal terms are indicated by the boxed matrices in Eqs. 3.7- 3.22. The shapes of the tranversitytransformed matrices are presented in Appendices C \& D.

\section{B. Phase class}

As discussed in Appendices C \& D, the shape of the $\widetilde{\Gamma}, \widetilde{\omega}$ allows us to group these matrices into "phase classes." As in the "Legendre classes" of the original matrices $\Gamma, \omega$, the shapes are defined by where nonzero entries appear in the matrix. The classification into: diagonal, antidiagonal, left parallelogram, right parallelogram; crossed, and polygon shapes is of significance in that these shapes select the contributing bilinear helicity products. For example, if in the product $\widetilde{\Gamma}^{\alpha} \widetilde{\omega}^{\beta}$ both matrices are diagonal, then that observable depends on linear combinations of the product: $\left|\widetilde{H}_{a, \lambda_{V}}\right|^{2}$. If on the other hand the matrix product $\widetilde{\Gamma}^{\alpha} \widetilde{\omega}^{\beta}$ has an entry at the location $a, b$ in the $\widetilde{\Gamma}$ space and at the location $\lambda_{V}, \lambda_{V}^{\prime}$ in the $\widetilde{\omega}$ space, then that observable depends on the following product:

$$
\widetilde{H}_{a, \lambda_{V}}^{*} \widetilde{H}_{b, \lambda_{V}^{\prime}}=\left|\widetilde{H}_{a, \lambda_{V}}\right|\left|\widetilde{H}_{b, \lambda_{V}^{\prime}}\right| \exp i\left(\phi_{b, \lambda_{V}^{\prime}}-\phi_{a, \lambda_{V}}\right),
$$

where $\widetilde{H}_{a, \lambda_{V}}=\left|\widetilde{H}_{a, \lambda_{V}}\right| \exp i\left(\phi_{a, \lambda_{V}}\right)$, etc. Thus, the shapes of the matrices in the tranversity description tell us which phases $\phi_{b, \lambda_{V}^{\prime}}-\phi_{a, \lambda_{V}} \equiv \phi_{\lambda_{V}^{\prime} \lambda_{V}}^{b, a}$ are needed to determine the associated spin observable. In Fig. 2, the basic problem of determining the twelve amplitudes is illustrated, where the lengths of the vectors correspond to the magnitude of the transversity amplitudes, and the phases of these complex amplitudes are also shown. To fix this diagram, we need to determine 12 magnitudes and then 11 phases; one overall phase and the overall orientation of the diagram of Fig. 2 is arbitrary. This situation is a generalization of the pseudoscalar case, which is described in Appendix E and by Fig. 3.

\section{Complete experiments}

The phase classification of the transversity matrices are a guide to the task of picking a complete set of 
experiments. Thus the procedure is to first select experiments which give information about the magnitudes of the 12 transversity amplitudes and then to pick experiments which yield nonredundant phase information. In the pseudoscalar case, the single spin observables yield the magnitudes of all four transversity amplitudes. Then three double spin observables, selected using the BDS 10,11] rules, yield three phases. The task is similar for the vector case, but the result is more complicated.

To extract the magnitudes of the transversity amplitudes, we need to examine all observables for which both $\widetilde{\Gamma}^{\alpha}$ and $\widetilde{\omega}^{\beta}$ are of the diagonal phase class. These observables are produced by all products: $\widetilde{\Gamma}^{1,4,10,12} \times \widetilde{\omega}^{1,3, A}$, see Appendix C and D.

In the vector meson case there are only eight independent single spin observables, so we learn that this case is not as favorable as the pseudoscalar meson case, where the single spin observables sufficed to determine all four amplitude magnitudes. Indeed, the situation is that only six of the vector meson photoproduction single spin observables are of diagonal form; namely, $\mathcal{I}, \check{T}, \check{P}_{N^{\prime}}, \check{\Sigma}, \check{P}_{V}$ and the tensor polarization $\check{T}_{y y}$. Thus we need to turn to the double spin observables for the remaining six diagonal phase class matrices reside. Therefore, for vector meson photoproduction it is not possible to determine the magnitudes of the twelve transversity amplitudes by only measuring six single spin observables.

\section{CONCLUSIONS}

Several conclusions can be drawn from describing spin observables for vector meson photoproduction in bilinear helicity product form. Here one essentially extends the BDS 10 11 rules to include the vector meson degree of freedom.

The diagonal matrices in the transversity basis are indicated by boxes in Eqs. 3.7-3.22. For the corresponding observables, the diagonal nature of the product $\widetilde{\Gamma}^{\alpha} \widetilde{\omega}^{\beta}$ means that those observables depend on linear combinations of the squared magnitude of the transversity amplitudes, e.g., they depend on combinations of $\pm\left|\widetilde{H}_{i, \lambda_{V}}\right|^{2}$.

Those experiments which depend only on the magnitude of the transversity amplitude are the following six single spin observables:

$$
\mathcal{I}, \check{T}, \check{P}_{N^{\prime}}, \check{\Sigma}, \check{P_{V}}, \check{T_{y y}}
$$

plus the following six double spin observables:

$$
\check{C}_{x y}^{\gamma N}, \check{C}_{x y}^{\gamma N^{\prime}}, \check{C}_{y y}^{N N^{\prime}}, \check{C}_{x y}^{\gamma V}, \check{C}_{y y}^{N V}, \check{C}_{y y}^{N^{\prime} V} .
$$

Note the above $x$-component for the photon beam corresponds to a photon linearly polarized perpendicular to the scattering plane [7]. In contrast to the pseudoscalar meson production case, the magnitudes of the transversity amplitudes for the vector meson case can not be determined by just single spin observable measurements, 
one needs to also perform six double spin measurements. Only six of the eight independent single spin observables are of diagonal form; the remaining two single spin observables provide transversity amplitude phase information. In addition, of those six double spin observables three involve measuring the spin state of the final vector meson (via its decay)

$$
\check{C}_{x y}^{\gamma V}, \check{C}_{y y}^{N V}, \check{C}_{y y}^{N^{\prime} V}
$$

two require a polarized photon beam

$$
\check{C}_{x y}^{\gamma N}, \check{C}_{x y}^{\gamma N^{\prime}}
$$

and one requires a polarized target and measurement of the spin state of the recoil final baryon

$$
\check{C}_{y y}^{N N^{\prime}}
$$

That sets the task for determining the magnitudes of the transversity amplitudes, which provide the most convenient situation. Note that near threshold, the two spin observables $\check{C}_{x y}^{\gamma V}$, and $\check{C}_{x y}^{\gamma N}$ vanish.

Observables for which both $\widetilde{\Gamma}^{\alpha}$ and $\widetilde{\omega}^{\beta}$ are not diagonal depend on transversity amplitude phase differences. As an extension of the BDS rules for the pseudoscalar meson case, these transversity phase-dependent observables form phase classes in which some experiments provide redundant phase information. A graphical procedure for analyzing the redundancy and phase class of experiments for vector meson production is illustrated in Fig. 2.

The procedure consists of picking a set of phases between transversity amplitudes that correspond to a given phase class for the $\Gamma$ and $\omega$ matrices. Then list all the $\Gamma \times \omega$ matrices of that same phase class. It is best to start with the two spin spin observables that are not diagonal in the transversity basis; for example $T_{20}, T_{21}$. The corresponding experiments can be selected from Tables II \& III, where only the number crresponding to the number of unknown phases need to be performed. In that process, some experiments prove to be feasible, some are difficult if not impossible; indeed, in some cases one needs to go to triple spin observables. In any event, this selection procedure can be used to answer the question of the experiments needed for a full determination of all phases and also which ones will be extremely difficult to determine because of realistic experimental conditions.

In addition to these features of using the bilinear helicity product form, one can deduce many other aspects of spin observables based on general knowledge of the properties of the $\Gamma^{\alpha} \omega^{\beta}$ matrices. For example, when a "Lorentz" $\left(S \gamma^{\mu} S^{-1}=\ell_{\nu}^{\mu} \gamma^{\nu}\right)$ transformation or a parity, chirality or time reversal operation is performed on the $\Gamma^{\alpha}$ and $\omega^{\beta}$ matrices in the "helicity amplitude space." they reveal linear relations between spin observables. If Fierz transformations are made on the $\Gamma^{\alpha}$ and $\omega^{\beta}$ matrices, again in helicity space, then quadratic relationships between spin observables are readily deduced. 
An important part of our analysis is the use of transversity amplitudes. Many other types of amplitudes can be defined. For example, one can use the unitary transformations from the Dirac $\gamma$-matrices to the chiral, Majorana or other $\gamma$-matrices sets to define new amplitudes; corresponding unitary transformations in the vector meson helicity 3 -space can also be invoked. Thus the amplitude basis is hardly unique and one can deduce other sets and therefore deduce other spin observables as the ones to measure to determine the magnitude and/or phases of these alternately defined amplitudes. However, there is something very special about the transversity amplitudes, which is closely related to using the normal to the scattering plan as the spin-quantization axis. The special property is that the transversity amplitudes make the optimum number of single spin observables independent of amplitude phases. Correspondingly, phase information is relegated to the more complicated spin observables. This is most dramatic for the four single spin observables for pseudoscalar meson photoproduction in that single spin observables provide the magnitude of all four transversity amplitudes. That simplification is also of benefit for vector mesons, which suggests that there are compelling reasons to use transversity amplitudes.

We hope that the procedure described here will be useful in ascertaining the information content and the nodal behavior of vector meson spin observables and in planning experiments. Measurement of all spin observables needed to fully determine the twelve amplitudes is probably not feasible, although it is good to know what is needed for that full task. Even without a full experimental determination of the photoproduction amplitudes, it is possible to extract useful dynamical information, which is not an uncommon situation in strong interaction physics.

\section{ACKNOWLEDGMENTS}

We wish to thank Drs. S. N. Yang, B. Saghai and C. Fasano for their helpful comments.

\section{APPENDIX A: BASIS MATRICES}

\section{Four $\times$ four-matrices}

The basic properties of the 16 Hermitian $\Gamma$ matrices are presented here. These are well-known matrices, except that we have made them Hermitian in order to generate real observables when used in the 4-dimensional part of our helicity space amplitudes. The basic properties are:

$$
\operatorname{Tr}\left[\Gamma^{\alpha} \Gamma^{\beta}\right]=4 \delta_{\alpha \beta} \quad \Gamma^{\alpha \dagger}=\Gamma^{\alpha}
$$

These permit one to expand any $4 \times 4$ matrix $X$ in terms of the $\Gamma$ 's and to extract the expansion coefficients $C_{\beta}$ using: $X=\sum_{\beta} C_{\beta} \Gamma^{\beta}$ and $C_{\beta}=\frac{1}{4} \operatorname{Tr}\left[\Gamma^{\beta} X\right]$. The 
definition of the $\Gamma$ matrices in terms of $\gamma^{\mu}, \gamma^{5}, \sigma^{\mu \nu} \ldots$ is presented in Eq. 3.3.

\section{Three $\times$ three-matrices}

The basic properties of the 9 Hermitian $\omega$ matrices are presented here. These are Hermitian in order to generate real observables when used in the 3-dimensional part of our helicity space amplitudes. The basic properties are:

$$
\operatorname{Tr}\left[\omega^{\alpha} \omega^{\beta}\right]=3 \delta_{\alpha \beta} \quad \omega^{\alpha \dagger}=\omega^{\alpha}
$$

As in the $4 \times 4$ case, these properties allow one to expand a general $3 \times 3$ matrix and to extract the associated expansion coefficients.

The nine $\omega$ matrices are defined in the text as a unit matrix, plus three vector spin-one matrices, plus a rank-2 tensor. As given earlier the Cartesian form of the rank-2, symmetric Hermitian tensor is:

$$
t_{i j}=\frac{S_{i} S_{j}+S_{j} S_{i}}{2}-\frac{2}{3} \delta_{i j}
$$

The associated spherical tensor form is:

$$
\begin{gathered}
t_{20}=\sqrt{\frac{3}{2}} \tau_{z z}=-\frac{1}{\sqrt{6}}\left(\omega^{8}+\omega^{9}\right) \\
t_{2 \pm 1}=\mp\left(t_{x z} \pm i t_{y z}\right)=\mp \frac{1}{\sqrt{6}}\left(\omega^{6} \pm i \omega^{7}\right) \\
t_{2 \pm 2}=\left(+\frac{t_{x x}-t_{y y}}{2} \pm i t_{x y}\right)=\frac{1}{\sqrt{3}}\left(\frac{\omega^{8}-\omega^{9}}{2}\right) \pm \frac{i}{\sqrt{6}} \omega^{5}
\end{gathered}
$$

where these are also expressed in terms of the Cartesian rank-2 tensor or the $\omega^{5-9}$ matrices.

The following diagonal terms are of particular interest:

$$
\begin{aligned}
& t_{y y}=-\frac{t_{22}+t_{2-2}}{2}-\frac{t_{20}}{\sqrt{6}}=S_{y}^{2}-\frac{2}{3}, \\
& t_{x x}=+\frac{t_{22}+t_{2-2}}{2}-\frac{t_{20}}{\sqrt{6}}=S_{x}^{2}-\frac{2}{3} .
\end{aligned}
$$

Here the matrix $t_{i j}$ is mapped to the rank 2 spherical tensor operator $t_{2 \mu}$; it is used in the BHP form for spin observables. The same rules apply to the operator $\tau_{2 \mu}$, which appears in the trace form for spin observables.

\section{APPENDIX B: ORIGINAL-BASIS MATRICES}

\section{Original four $\times$ four}

The sixteen $\Gamma$ matrices can be grouped into four classes with four members in each class according to 
their "shape." By shape, we mean the location of nonzero entries. For the original(Dirac) Hermitian matrices the shapes are: diagonal $(D)$; antidiagonal $(A D)$; left parallelogram $(P L)$; and right parallelogram $(P R)$. The first Legendre class is of diagonal shape $(D)$ and has $\Gamma^{1,2,9,15}$ as its members:

$$
\Gamma_{D}=\left[\begin{array}{llll}
a & 0 & 0 & 0 \\
0 & b & 0 & 0 \\
0 & 0 & c & 0 \\
0 & 0 & 0 & d
\end{array}\right] \begin{array}{ccccc} 
& a & b & c & d \\
\Gamma_{1} & +1 & +1 & +1 & +1 \\
\Gamma_{2} & +1 & +1 & -1 & -1 \\
\Gamma_{9} & +1 & -1 & +1 & -1 \\
\Gamma_{15} & -1 & +1 & +1 & -1
\end{array} .
$$

The second Legendre class is of antidiagonal shape $(A D)$ and has $\Gamma^{3,4,6,7}$ as its members:

$$
\Gamma_{A D}=\left[\begin{array}{cccc}
0 & 0 & 0 & a \\
0 & 0 & b & 0 \\
0 & c & 0 & 0 \\
d & 0 & 0 & 0
\end{array}\right] ; \begin{array}{ccccc}
a & b & c & d \\
\Gamma_{3} & +i & +i & -i & -i \\
\Gamma_{4} & +1 & -1 & -1 & +1 \\
\Gamma_{6} & -1 & -1 & -1 & -1 \\
\Gamma_{7} & +i & -i & +i & -i
\end{array} .
$$

The third Legendre class is of left parallelogram shape $(P L)$ and has $\Gamma^{10,11,13,14}$ as its members:

$$
\Gamma_{P L}=\left[\begin{array}{llll}
0 & a & 0 & 0 \\
b & 0 & 0 & 0 \\
0 & 0 & 0 & c \\
0 & 0 & d & 0
\end{array}\right] \begin{array}{ccccc}
a & b & c & d \\
\Gamma_{10} & +i & -i & +i & -i \\
\Gamma_{11} & -1 & -1 & -1 & -1 \\
\Gamma_{13} & -1 & -1 & +1 & +1 \\
\Gamma_{14} & +i & -i & -i & +i
\end{array}
$$

The third Legendre class is of right parallelogram shape $(P R)$ and has $\Gamma^{10,11,13,14}$ as its members:

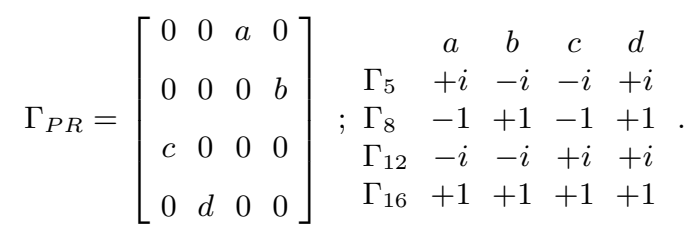

These shapes are important for the determination of the angular dependence of spin observables, e.g., their nodal structure.

Later, the associated shapes for the transversity transformed matrices $\widetilde{\Gamma}$ will be presented, which are useful for the analysis of a complete set of experiments.

\section{Original three $\times$ three}

The nine $\omega$ matrices can also be grouped into four classes according to their "shape." By shape, we mean the location of nonzero entries. For the original Hermitian matrices, the shapes are: diagonal $(D)$; antidiagonal $(A D)$; diamond or polygon $(P)$; and $\operatorname{crossed}(X)$. In this case there are two $D$, one $A D$, four $P$, and two $X$ matrices, which accounts for the nine $\omega^{\beta}$ matrices. In addition, we classify the three matrices $\omega^{A, B, C}$, which are particular linear combinations of 
$\omega^{1,8,9}$, see Eqs. 3.17-3.16. The matrix $\omega^{A}$ is antidiagonal; whereas $\omega^{B}$ and $\omega^{C}$ are of crossed $X$ shape. The explicit forms are:

$$
\begin{aligned}
& \omega_{D}=\left[\begin{array}{ccc}
a & 0 & 0 \\
0 & b & 0 \\
0 & 0 & c
\end{array}\right] \quad \begin{array}{cccc}
a & b & c \\
\omega 1 & +1 & +1 & +1 \\
\omega 4 & \frac{\sqrt{3}}{2} & 0 & -\frac{\sqrt{3}}{2}
\end{array} \\
& \omega_{A D}=\left[\begin{array}{ccc}
0 & 0 & a \\
0 & b & 0 \\
c & 0 & 0
\end{array}\right] ; \begin{array}{cccc} 
& a & b & c \\
\omega A & -i \frac{\sqrt{6}}{2} & 0 & +i \frac{\sqrt{6}}{2} \\
\omega A & -1 & +1 & -1
\end{array} \text {. }
\end{aligned}
$$

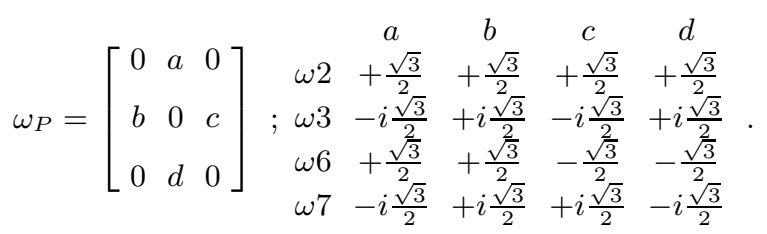

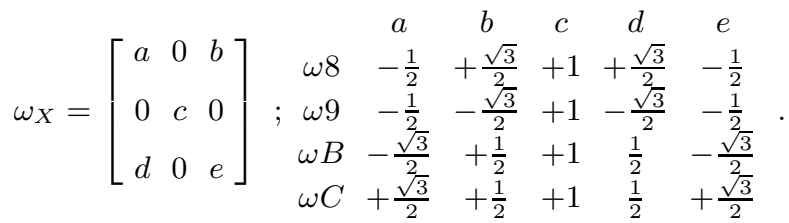

The classification of these matrices are helpful in the analysis of the angular dependence of spin observables, e.g., of the "Legendre class" and the associated nodal structure. The associated shapes for the transversity transformed matrices $\widetilde{\omega}$, which are useful for the analysis of a complete set of experiments, will be presented later.

\section{APPENDIX C: TRANSVERSITY-BASIS MATRICES}

Introducing the transversity amplitudes involves a unitary transformation of the basis matrices, see Eqs. 5.1 5.4. These transformed matrices are presented here for the $4 \times 4, \widetilde{\Gamma}$ and $3 \times 3, \widetilde{\omega}$ cases.

\section{Transversity four $\times$ four}

After the transversity transformation, the sixteen $\widetilde{\Gamma}$ matrices still form four classes, with four members in each class. Since these matrices are part of the analysis of which experiments are needed to determine the magnitude and phase of the transversity amplitudes, we refer to these as the "phase class."

The first phase class is of diagonal shape $(D)$ and has $\widetilde{\Gamma}^{1,4,10,12}$ as its members:

$$
\widetilde{\Gamma}_{D}=\left[\begin{array}{llll}
a & 0 & 0 & 0 \\
0 & b & 0 & 0 \\
0 & 0 & c & 0 \\
0 & 0 & 0 & d
\end{array}\right] ; \begin{array}{ccccc} 
& \\
\widetilde{\Gamma}_{1} & +1 & +1 & +1 & +1 \\
\widetilde{\Gamma}_{10} & -1 & +1 & -1 & -1 \\
\widetilde{\Gamma}_{12} & -1 & +1 & -1 & +1
\end{array} .
$$


The second phase class is of antidiagonal shape $A(D)$ and has $\widetilde{\Gamma}^{2,7,14,16}$ as its members:

$$
\widetilde{\Gamma}_{A D}=\left[\begin{array}{cccc}
0 & 0 & 0 & a \\
0 & 0 & b & 0 \\
0 & c & 0 & 0 \\
d & 0 & 0 & 0
\end{array}\right] ; \begin{array}{ccccc} 
& a & b & c & d \\
\widetilde{\Gamma}_{2} & +1 & +1 & +1 & +1 \\
\widetilde{\Gamma}_{7} & -i & -i & +i & +i \\
\widetilde{\Gamma}_{14} & -1 & +1 & +1 & -1 \\
\widetilde{\Gamma}_{16} & +i & -i & +i & -i
\end{array} .
$$

The third phase class is of left parallelogram shape $(P L)$ and has $\widetilde{\Gamma}^{6,8,13,15}$ as its members:

$$
\widetilde{\Gamma}_{P L}=\left[\begin{array}{cccc}
0 & a & 0 & 0 \\
b & 0 & 0 & 0 \\
0 & 0 & 0 & c \\
0 & 0 & d & 0
\end{array}\right] ; \begin{array}{ccccc} 
& a & b & c & d \\
\widetilde{\Gamma}_{6} & -1 & -1 & +1 & +1 \\
\widetilde{\Gamma}_{8} & -i & +i & -i & +i \\
\widetilde{\Gamma}_{13} & +i & -i & -i & +i \\
\widetilde{\Gamma}_{15} & -1 & -1 & -1 & -1
\end{array} .
$$

The fourth phase class is of right parallelogram shape $(P R)$ and has $\widetilde{\Gamma}^{3,5,9,11}$ as its members:

$$
\widetilde{\Gamma}_{P R}=\left[\begin{array}{cccc}
0 & 0 & a & 0 \\
0 & 0 & 0 & b \\
c & 0 & 0 & 0 \\
0 & d & 0 & 0
\end{array}\right] ; \begin{array}{ccccc} 
& a & b & c & d \\
\widetilde{\Gamma}_{3} & -i & -i & +i & +i \\
\widetilde{\Gamma}_{5} & +1 & -1 & +1 & -1 \\
\widetilde{\Gamma}_{9} & +1 & +1 & +1 & +1 \\
\widetilde{\Gamma}_{11} & +i & -i & -i & +i
\end{array} .
$$

\section{APPENDIX D: TRANSVERSITY THREE $\times$ THREE}

After the transversity transformation, the nine $\widetilde{\omega}$ matrices still form four classes. For these transversity Hermitian matrices, the shapes are still: diagonal $(D)$; antidiagonal $(A D)$; diamond or polygon $(P)$; and $\operatorname{crossed}(X)$. In this case there are two $D$, one $A D$, four $P$, and two $X$ matrices, which accounts for the nine $\widetilde{\omega}^{\beta}$ matrices. In addition, we classify the three matrices $\widetilde{\omega}^{A, B, C}$, which are particular linear combinations of $\widetilde{\omega}^{1,8,9}$, see Eqs. 3.17 3.16. The matrix $\widetilde{\omega}^{A}$ is now diagonal; whereas $\widetilde{\omega}^{B}$ and $\widetilde{\omega}^{C}$ are of crossed $X$ shape.

The first phase class is of diagonal shape $(D)$ and has $\widetilde{\omega}^{1,3, A}$ as its members:

$$
\widetilde{\omega}_{D}=\left[\begin{array}{lll}
a & 0 & 0 \\
0 & b & 0 \\
0 & 0 & c
\end{array}\right] ; \begin{array}{cccc} 
& a & b & c \\
\widetilde{\omega}^{1} & +1 & +1 & +1 \\
\widetilde{\omega}^{3}+\frac{\sqrt{6}}{2} & 0 & -\frac{\sqrt{6}}{2} \\
\widetilde{\omega}^{A} & +1 & -1 & +1
\end{array} .
$$

The second phase class is of antidiagonal shape $(A D)$ and has $\widetilde{\omega}^{6}$ as its sole member:

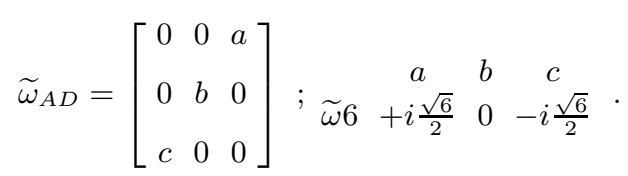


The third phase class is of polygon shape $(P)$ and has $\widetilde{\omega}^{2,4,5,7}$ as its four members:

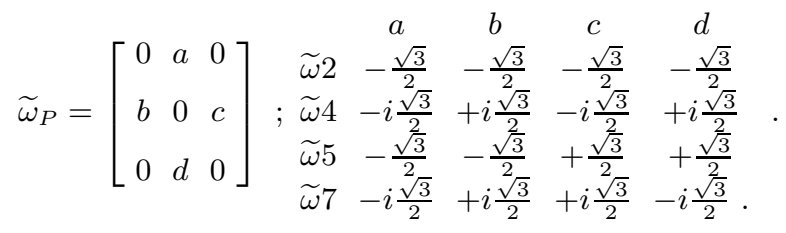

The fourth phase class is of crossed shape $(X)$ and has $\widetilde{\omega}^{8,9, B, C}$ as its four members:

$\widetilde{\omega}_{X}=\left[\begin{array}{ccc}a & 0 & b \\ 0 & c & 0 \\ d & 0 & e\end{array}\right] ; \begin{array}{ccccccc}\widetilde{\omega} 8 & \frac{1-\sqrt{3}}{4} & \frac{3+\sqrt{3}}{4} & -\frac{1-\sqrt{3}}{2} & \frac{3+\sqrt{3}}{4} & \frac{1-\sqrt{3}}{4} \\ & \widetilde{\omega} 9 & \frac{1+\sqrt{3}}{4} & \frac{3-\sqrt{3}}{4} & -\frac{1+\sqrt{3}}{2} & \frac{3-\sqrt{3}}{4} & \frac{1+\sqrt{3}}{4} \\ \widetilde{\omega} B & \frac{1-\sqrt{3}}{4} & \frac{3+\sqrt{3}}{4} & +\frac{1-\sqrt{3}}{2} & \frac{3+\sqrt{3}}{4} & \frac{1-\sqrt{3}}{4} \\ & \widetilde{\omega} C & \frac{1+\sqrt{3}}{4} & \frac{3-\sqrt{3}}{4} & +\frac{1+\sqrt{3}}{2} & \frac{3-\sqrt{3}}{4} & \frac{1+\sqrt{3}}{4}\end{array}$.

These phase classes are useful in specifying the experiments needed to determine the magnitude and phases of the 12 complex transversity amplitudes.

\section{APPENDIX E: PSEUDOSCALAR MESONS}

We can return to the case of pseudoscalar meson photoproduction by omitting all vector meson spin observables and by replacing all $\omega$ matrices by zero, except for the $\omega^{1, A} \rightarrow 1$ case. In addition, the twelve amplitudes reduce to four: $H_{a, \lambda_{V}} \rightarrow H_{a}$. This limit is equivalent to looking at the $\lambda_{V} \rightarrow 0$ terms only. For the pseudoscalar meson case, we next present the BHP spin observable profiles.

\section{Single spin observables}

There are four single spin observables for pseudoscalar meson photoproduction, where we include the crosssection:

$$
\begin{aligned}
\text { CROSS - SECTION : } I & =\frac{1}{2}<H \| \Gamma^{1} \mid H> \\
\text { TARGET : } \check{T}= & -\frac{1}{2}<H \sqrt{\Gamma^{10}} \mid H> \\
\text { RECOIL : } \check{P}_{N^{\prime}} & =\frac{1}{2}<H \| \Gamma^{12} \mid H> \\
\text { BEAM : } \check{\Sigma} & =\frac{1}{2}<H \| \Gamma^{4} \mid H>
\end{aligned}
$$

Note that all of these single spin observables are diagonal in the transversity amplitude case, which is the meaning of the boxed $\Gamma$ matrices. Therefore, measurement of the four spin observables yields the magnitudes of all four transversity amplitudes $\widetilde{H}_{1} \cdots \widetilde{H}_{4}$. To determine the amplitude phases, one needs to measure double spin observables. 


\section{Double spin observables}

There are four transversity amplitudes and hence four amplitude phases; however, one overall phase is arbitrary. Therefore, one needs to perform three measurements to fix these three phases, see Fig. 3.

$$
\begin{gathered}
\text { BEAM - TARGET : } \check{C}_{i j}^{\gamma N}=\frac{1}{2}\left\langle H\left|\mathcal{C}_{i j}^{\gamma N}\right| H\right\rangle \\
\mathcal{C}^{\gamma N}=\left(\begin{array}{ccc}
0 & -\Gamma^{12} & 0 \\
\Gamma^{5} & 0 & \Gamma^{3} \\
\Gamma^{11} & 0 & \Gamma^{9}
\end{array}\right)=\left(\begin{array}{ccc}
0 & -P_{N^{\prime}} & 0 \\
H & 0 & G \\
F & 0 & E
\end{array}\right)
\end{gathered}
$$

BEAM - RECOIL : $\check{C}_{i j}^{\gamma N^{\prime}}=\frac{1}{2}<H\left|\mathcal{C}_{i j}^{\gamma N^{\prime}}\right| H>$

$\mathcal{C}^{\gamma N^{\prime}}=\left(\begin{array}{ccc}0 & \Gamma^{10} & 0 \\ \Gamma^{14} & 0 & -\Gamma^{7} \\ -\Gamma^{16} & 0 & -\Gamma^{2}\end{array}\right)=\left(\begin{array}{ccc}0 & -T & 0 \\ O_{x^{\prime}} & 0 & O_{z^{\prime}} \\ C_{x^{\prime}} & 0 & C_{z^{\prime}}\end{array}\right)$

TARGET - RECOIL : $\check{C}_{i j}^{N N^{\prime}}=\frac{1}{2}<H\left|\mathcal{C}_{i j}^{N N^{\prime}}\right| H>$

$\mathcal{C}^{N N^{\prime}}=\left(\begin{array}{ccc}-\Gamma^{6} & 0 & -\Gamma^{13} \\ 0 & -\Gamma^{4} & 0 \\ \Gamma^{8} & 0 & \Gamma^{15}\end{array}\right)=\left(\begin{array}{ccc}T_{x^{\prime}} & 0 & T_{z^{\prime}} \\ 0 & -\Sigma & 0 \\ L_{x^{\prime}} & 0 & L_{z^{\prime}}\end{array}\right)$

The three boxed $\Gamma$ matrices in the above double spin observables, already appeared in the single spin observables. Thus there are only four, instead of five, members in each of the above double spin categories. In particular, the following double spin observables are equal to single spin observables:

$$
\mathcal{C}_{x y}^{\gamma N}=-P_{N^{\prime}} \quad \mathcal{C}_{x y^{\prime}}^{\gamma N^{\prime}}=-T \mathcal{C}_{y y^{\prime}}^{N N^{\prime}}=-\Sigma .
$$

These equalities are direct consequences of parity conservation. For the vector meson case, the appearance of the $\omega^{A}$ matrix in the corresponding double spin observables yields linear relations between observables, not the above restriction; hence, in that case there are five double spin observables in these categories.

In addition, the $\Gamma^{\alpha}$ matrices for each double spin observable are of the same "phase class." For $\mathcal{C}^{\gamma N} \alpha=$ 3, 5, 9, 11 appear, which are of phase class $P R$. Based on this shape category, these observables depend on the following relative phases: $\phi_{1,3}, \phi_{2,4}$. For $\mathcal{C}^{\gamma N^{\prime}} \alpha=2,7,14,16$ appear, which are of phase class $A D$. Based on this shape category, these observables depend on the following relative phases: $\phi_{1,4}, \phi_{2,3}$. For $\mathcal{C}^{N N^{\prime}} \alpha=6,8,13.15$ appear, which are of phase class $P L$. Based on this shape category,these observables depend on the following relative phases: $\phi_{1,2}, \phi_{3,4}$. Here, we label the phases using $\phi_{a, b}$ for the phase difference $\phi_{a, b}=\phi_{a}-\phi_{b}$ between the transversity amplitudes $\widetilde{H}_{a}$ and $\widetilde{H}_{b}$, see Fig. 3 . 
Making three measurements of the same phase class observables would be redundant; only two are needed for the two phases. The third measurement should be taken from another phase class. Thus one needs three double spin measurements, but not more than two from a given phase class. Here we have presented a derivation of the BDS [10]11] rules, based on the shape of the $\widetilde{\Gamma}$ matrices and the geometric picture of the transversity amplitudes shown in Fig. 3. The advantage of this rendition of the BDS theorem is that it can be generalized to the case of vector meson photoproduction, see Fig.2.

\section{Triple spin observables}

In the pseudoscalar meson case, the following triple spin observables can be derived in the BHP form. Again, the triple spin observables are displayed in a Cartesian format with:

$$
\begin{aligned}
& \check{C}_{x i j}^{\gamma N N^{\prime}}=\frac{1}{2}<H\left|\mathcal{C}_{i j}^{\gamma N N^{\prime}}\right| H>, \\
& \check{C}_{y i j}^{\gamma N N^{\prime}}=\frac{1}{2}<H\left|\mathcal{C}_{y i j}^{\gamma N N^{\prime}}\right| H>, \\
& \check{C}_{z i j}^{\gamma N N^{\prime}}=\frac{1}{2}<H\left|\mathcal{C}_{z i j}^{\gamma N N^{\prime}}\right| H>, \\
& \mathcal{C}_{x i j}^{\gamma N N^{\prime}}=\left(\begin{array}{ccc}
\Gamma^{15} & 0 & -\Gamma^{8} \\
0 & -\Gamma^{1} & 0 \\
\Gamma^{13} & 0 & -\Gamma^{6}
\end{array}\right)=\left(\begin{array}{ccc}
-L_{z^{\prime}} & 0 & L_{x^{\prime}} \\
0 & -I & 0 \\
T_{z^{\prime}} & 0 & -T_{x^{\prime}}
\end{array}\right) \\
& \mathcal{C}_{y i j}^{\gamma N N^{\prime}}=\left(\begin{array}{ccc}
0 & -\Gamma^{9} & 0 \\
-\Gamma^{2} & 0 & \Gamma^{16} \\
0 & -\Gamma^{11} & 0
\end{array}\right)=\left(\begin{array}{ccc}
0 & -E & 0 \\
C_{z^{\prime}} & 0 & -C_{x^{\prime}} \\
0 & F & 0
\end{array}\right) \\
& \mathcal{C}_{z i j}^{\gamma N N^{\prime}}=\left(\begin{array}{ccc}
0 & -\Gamma^{3} & 0 \\
\Gamma^{7} & 0 & \Gamma^{14} \\
0 & \Gamma^{5} & 0
\end{array}\right)=\left(\begin{array}{ccc}
0 & G & 0 \\
-O_{z^{\prime}} & 0 & O_{x^{\prime}} \\
0 & H & 0
\end{array}\right) .
\end{aligned}
$$

Here all three particles with spin are involved. Of the $3 \times 9$ possible triple spin observables, 15 are nonzero. Three of these are equal to spin spin observables and the remaining 12 are equal to double spin observables. Therefore, there is no new information in triple spin observables for pseudoscalar meson photoproduction, and it is fortunately not necessary to consider such complicated measurements. This is not the case for vector meson photoproduction.

\section{APPENDIX F: VECTOR MESONS}

The single and double spin observables were presented in the text. Using the BHP approach and MAPLE, it is 
possible to derive explicit expressions for the triple and quadruple spin observables for vector meson photoproduction. Triple spin observables involve the spin of three particles, including the vector and tensor polarization of the vector meson.

\section{Triple spin observables}

There are four types of triple spin observables. The first three types, $N, N^{\prime}, V, \gamma, N^{\prime}, V$, and $\gamma, N, V$ involve the vector meson. The fourth type involves the $\gamma, N, N^{\prime}$ particles and does not include the vector meson:

$$
\begin{aligned}
& \mathcal{C}_{x i j}^{N N^{\prime} V}=\left(\begin{array}{lll}
\Gamma^{6} & -\Gamma^{3} & \Gamma^{13}
\end{array}\right)\left(\begin{array}{ccccccccc}
0 & \omega^{3} & 0 & 0 & \omega^{6} & 0 & \omega^{8} & \omega^{9} \\
\omega^{2} & 0 & \omega^{4} & \omega^{5} & 0 & \omega^{7} & 0 & 0 \\
0 & \omega^{3} & 0 & 0 & \omega^{6} & 0 & \omega^{8} & \omega^{9}
\end{array}\right), \\
& \mathcal{C}_{y i j}^{N N^{\prime} V}=\left(\begin{array}{ll}
\Gamma^{7} & -\Gamma^{4} \Gamma^{14}
\end{array}\right)\left(\begin{array}{cccccccc}
\omega^{2} & 0 & \omega^{4} & \omega^{5} & 0 & \omega^{7} & 0 & 0 \\
0 & \omega^{3} & 0 & 0 & \omega^{6} & 0 & \omega^{8} & \omega^{9} \\
\omega^{2} & 0 & \omega^{4} & \omega^{5} & 0 & \omega^{7} & 0 & 0
\end{array}\right), \\
& \left.\mathcal{C}_{z i j}^{N N^{\prime} V}=\begin{array}{lll}
\Gamma^{8} & -\Gamma^{5} & \Gamma^{15}
\end{array}\right)\left(\begin{array}{cccccccc}
0 & \omega^{3} & 0 & 0 & \omega^{6} & 0 & \omega^{8} & \omega^{9} \\
\omega^{2} & 0 & \omega^{4} & \omega^{5} & 0 & \omega^{7} & 0 & 0 \\
0 & \omega^{3} & 0 & 0 & \omega^{6} & 0 & \omega^{8} & \omega^{9}
\end{array}\right) .
\end{aligned}
$$

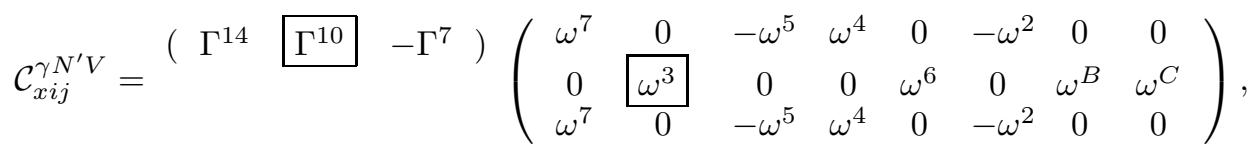

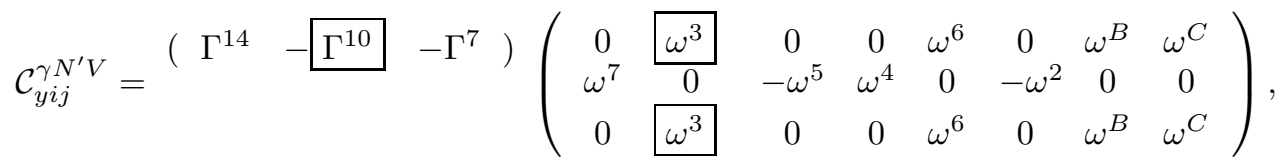

$$
\begin{aligned}
& \mathcal{C}_{z i j}^{\gamma N^{\prime} V}=-\left(\begin{array}{ll}
\Gamma^{16} & -\Gamma^{12} \\
& \Gamma^{2}
\end{array}\right)\left(\begin{array}{cccccccc}
0 & \omega^{3} & 0 & 0 & \omega^{6} & 0 & \omega^{8} & \omega^{9} \\
\omega^{2} & 0 & \omega^{4} & \omega^{5} & 0 & \omega^{7} & 0 & 0 \\
0 & \omega^{3} & 0 & 0 & \omega^{6} & 0 & \omega^{8} & \omega^{9}
\end{array}\right) . \\
& \mathcal{C}_{x i j}^{\gamma N V}=-\left(\begin{array}{ll}
\Gamma^{5} & \Gamma^{12} \\
& \Gamma^{3}
\end{array}\right)\left(\begin{array}{cccccccc}
\omega^{7} & 0 & -\omega^{5} & \omega^{4} & 0 & -\omega^{2} & 0 & 0 \\
0 & \omega^{3} & 0 & 0 & \omega^{6} & 0 & \omega^{B} & \omega^{C} \\
\omega^{7} & 0 & -\omega^{5} & \omega^{4} & 0 & -\omega^{2} & 0 & 0
\end{array}\right), \\
& \mathcal{C}_{y i j}^{\gamma N V}=-\left(\begin{array}{ll}
\Gamma^{5} & -\Gamma^{12} \\
\Gamma^{3}
\end{array}\right)\left(\begin{array}{cccccccc}
0 & \omega^{3} & 0 & 0 & \omega^{6} & 0 & \omega^{B} & \omega^{C} \\
\omega^{7} & 0 & -\omega^{5} & \omega^{4} & 0 & -\omega^{2} & 0 & 0 \\
0 & \omega^{3} & 0 & 0 & \omega^{6} & 0 & \omega^{B} & \omega^{C}
\end{array}\right),
\end{aligned}
$$

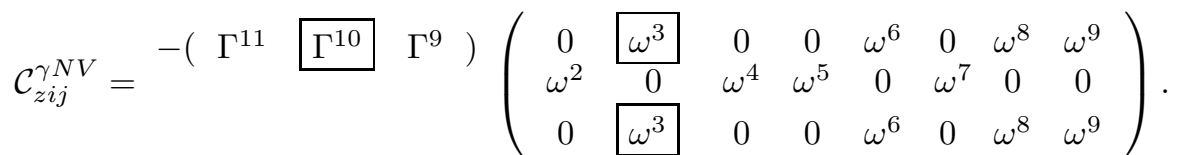




$$
\begin{aligned}
\mathcal{C}_{x i j}^{\gamma N N^{\prime}} & =\left(\begin{array}{ccc}
\Gamma^{15} & 0 & -\Gamma^{8} \\
0 & -\Gamma^{1} & 0 \\
\Gamma^{13} & 0 & -\Gamma^{6}
\end{array}\right) \omega^{A}, \\
\mathcal{C}_{y i j}^{\gamma N N^{\prime}} & =\left(\begin{array}{ccc}
0 & -\Gamma^{9} & 0 \\
-\Gamma^{2} & 0 & \Gamma^{16} \\
0 & -\Gamma^{11} & 0
\end{array}\right) \omega^{A}, \\
\mathcal{C}_{z i j}^{\gamma N N^{\prime}} & =\left(\begin{array}{ccc}
0 & -\Gamma^{3} & 0 \\
\Gamma^{7} & 0 & \Gamma^{14} \\
0 & \Gamma^{5} & 0
\end{array}\right) \cdot \omega^{1}
\end{aligned}
$$

\section{Quadruple spin observables}

Quadruple spin observables involve the spin of all four particles, including the vector and tensor polarization of the vector meson. Of course all of these involve the vector meson, including its vector and tensor polarization; hence, the $3 \times 8$ Cartesian display appears again. The question arises: Are all of the quadruple spin-observables redundant? In the pseudoscalar meson case the full case of triple spin observables were redundant in that they were all determined by single and/or double spin observable measurements. In the vector meson case, we have:

$$
\begin{aligned}
& \mathcal{C}_{x x i j}^{\gamma N N^{\prime} V}=\left(\begin{array}{ccc}
\Gamma^{15} & -\Gamma^{9} & -\Gamma^{8}
\end{array}\right)\left(\begin{array}{cccccccc}
0 & \omega^{3} & 0 & 0 & \omega^{6} & 0 & \omega^{B} & \omega^{C} \\
\omega^{7} & 0 & -\omega^{5} & \omega^{4} & 0 & -\omega^{2} & 0 & 0 \\
0 & \omega^{3} & 0 & 0 & \omega^{6} & 0 & \omega^{B} & \omega^{C}
\end{array}\right), \\
& \mathcal{C}_{x y i j}^{\gamma N N^{\prime} V}=-\left(\Gamma^{2} \Gamma^{1} \Gamma^{16}\right)\left(\begin{array}{ccccccccc}
\omega^{7} & 0 & -\omega^{5} & \omega^{4} & 0 & -\omega^{2} & 0 & 0 \\
0 & \omega^{3} & 0 & 0 & \omega^{6} & 0 & \omega^{B} & \omega^{C} \\
\omega^{7} & 0 & -\omega^{5} & \omega^{4} & 0 & -\omega^{2} & 0 & 0
\end{array}\right), \\
& \mathcal{C}_{x z i j}^{\gamma N N^{\prime} V}=\left(\begin{array}{ccc}
\Gamma^{13} & -\Gamma^{11} & -\Gamma^{6}
\end{array}\right)\left(\begin{array}{cccccccc}
0 & \omega^{3} & 0 & 0 & \omega^{6} & 0 & \omega^{B} & \omega^{C} \\
\omega^{7} & 0 & -\omega^{5} & \omega^{4} & 0 & -\omega^{2} & 0 & 0 \\
0 & \omega^{3} & 0 & 0 & \omega^{6} & 0 & \omega^{B} & \omega^{C}
\end{array}\right), \\
& \mathcal{C}_{y x i j}^{\gamma N N^{\prime} V}=\left(\begin{array}{lll}
-\Gamma^{15} & \Gamma^{9} & \Gamma^{8}
\end{array}\right)\left(\begin{array}{ccccccccc}
\omega^{7} & 0 & -\omega^{5} & \omega^{4} & 0 & -\omega^{2} & 0 & 0 \\
0 & \omega^{3} & 0 & 0 & \omega^{6} & 0 & \omega^{B} & \omega^{C} \\
\omega^{7} & 0 & -\omega^{5} & \omega^{4} & 0 & -\omega^{2} & 0 & 0
\end{array}\right), \\
& \mathcal{C}_{y y i j}^{\gamma N N^{\prime} V}=\left(\begin{array}{ll}
-\Gamma^{2} & \Gamma^{1} \\
\Gamma^{16}
\end{array}\right)\left(\begin{array}{cccccccc}
0 & \omega^{3} & 0 & 0 & \omega^{6} & 0 & \omega^{B} & \omega^{C} \\
\omega^{7} & 0 & -\omega^{5} & \omega^{4} & 0 & -\omega^{2} & 0 & 0 \\
0 & \omega^{3} & 0 & 0 & \omega^{6} & 0 & \omega^{B} & \omega^{C}
\end{array}\right), \\
& \mathcal{C}_{y z i j}^{\gamma N N^{\prime} V}=\left(\begin{array}{lll}
-\Gamma^{13} & -\Gamma^{11} & \Gamma^{6}
\end{array}\right)\left(\begin{array}{ccccccccc}
\omega^{7} & 0 & -\omega^{5} & \omega^{4} & 0 & -\omega^{2} & 0 & 0 \\
0 & \omega^{3} & 0 & 0 & \omega^{6} & 0 & \omega^{B} & \omega^{C} \\
\omega^{7} & 0 & -\omega^{5} & \omega^{4} & 0 & -\omega^{2} & 0 & 0
\end{array}\right),
\end{aligned}
$$




$$
\begin{gathered}
\mathcal{C}_{z x i j}^{\gamma N N^{\prime} V}= \\
\mathcal{C}_{z y i j}^{\gamma N N^{\prime} V}= \\
= \\
\left.\begin{array}{lll}
\Gamma^{7} & -\Gamma^{3} & \Gamma^{13}
\end{array}\right)\left(\begin{array}{cccccccc}
\omega^{2} & 0 & \omega^{4} & \omega^{5} & 0 & \omega^{7} & 0 & 0 \\
0 & \omega^{3} & 0 & 0 & \omega^{6} & 0 & \omega^{8} & \omega^{9} \\
\omega^{2} & 0 & \omega^{4} & \omega^{5} & 0 & \omega^{7} & 0 & 0
\end{array}\right), \\
\mathcal{C}_{z z i j}^{\gamma N N^{\prime} V}=\left(\begin{array}{ccccccccc}
0 & \omega^{3} & 0 & 0 & \omega^{6} & 0 & \omega^{8} & \omega^{9} \\
\omega^{2} & 0 & \omega^{4} & \omega^{5} & 0 & \omega^{7} & 0 & 0 \\
0 & \omega^{3} & 0 & 0 & \omega^{6} & 0 & \omega^{8} & \omega^{9}
\end{array}\right), \\
\left.\begin{array}{lllllllll}
-\Gamma^{8} & \Gamma^{5} & -\Gamma^{15}
\end{array}\right)
\end{gathered}
$$

* $\quad$ Research supported in part by the NSF.

[1] J. T. Dakin, G. J. Feldman, W. L. Lakin, F. Martin, Martin L. Perl, E. W. Petraske, and W. T. Toner, Phys.Rev.Lett.30(1973)142.

[2] J.M. Laget and R. Mendez-Galain, Nucl.Phys. A581 (1995) 397.

[3] E. M. Henley and A. G. Williams, Phys. Letters B281 (1992)178.

[4] E. M. Henley, G. Krein, J. Pollock, and A. G. Williams, Phys. Letters B269 (1991)31.

[5] A. I. Titov, Yongseok Oh, and Shin Nan Yang, "Electroproduction of $\phi$ Meson from Proton within Relativistic Harmonic Oscillator Model," 1st International Symposium on Symmetries in Subatomic Physics, Taiwan, May 1994.

[6] Frank Tabakin, Robert A. Eisenstein, Yang Lu, Phys. Rev. C44, (1991)1749.

[7] C. G. Fasano, Frank Tabakin, Bijan Saghai, Phys. Rev. C46, (1992)2430.

[8] Bijan Saghai and Frank Tabakin, "Nodal trajectories of spin observables and kaon photoproduction dynamics," preprint, 1995.

[9] R. L. Walker, Phys. Rev. 182 (1969) 1729.

[10] I. S. Barker, A. Donnachie, and J. K. Storrow, Nucl. Phys. B79 (1974)431

[11] I. S. Barker, A. Donnachie, and J. K. Storrow, Nucl. Phys. B95 (1975)347.

[12] M. Simonius, Phys. Rev. Letters 19 (1967) 279.

[13] H.E. Conzett, Rep. Prog. Phys. 57 (1994) 1-37.

[14] G. R. Goldstein, J. F. Owens III, J. P. Rutherfoord and M. J. Moravcsik, Nucl. Phys. B80 (1974)164.

[15] M. Jacob and G. C. Wick, Annals of Physics 7 (1959) 404.

[16] Çetin Şavkl, F. Tabakin and S. N. Yang, "Multipole Analysis of Spin Observables in Vector Meson Photoproduction," preprint, 1995. 
FIG. 1. The coordinate system and kinematical variables for vector meson photoproduction. Here V denotes the vector meson and $\lambda_{V}$ its helicity.

FIG. 2. This diagram displays the magnitudes and selected relative phase angles of the twelve transversity amplitudes $\widetilde{H}_{1 \lambda_{V}} \cdots \widetilde{H}_{4 \lambda_{V}}$ (with $\lambda_{V}=0, \pm 1$ ) for photoproduction of vector mesons. The twelve complex amplitudes are determined within an overall phase if the twelve magnitudes and eleven angles are fixed by 23 appropriate measurements. The magnitudes are determined by measuring six single spin observables plus six double spin observables, see the text. Then independent phase angle measurements are made by selecting spin observables from different "phase class" observables. The phase angles are labeled by the convention $\phi_{\lambda_{V} \lambda_{V}^{\prime}}^{s t}$

FIG. 3. This diagram displays the magnitudes and selected relative phase angles of the four transversity amplitudes $\widetilde{H}_{1} \cdots \widetilde{H}_{4}$ for photoproduction of pseudoscalar mesons. The four complex amplitudes are determined within an overall phase if the four magnitudes and three angles are fixed by seven appropriate measurements. The magnitudes are determined by measuring the four single spin observables, then independent phase angle measurements are made by selecting three double spin observables from at least two different "phase class" observables. The phase angles are labeled by the convention $\phi^{\text {st }}$ 
TABLE II. Spin Observables in BHP form as products of $\Gamma^{\alpha}$, and $\omega^{\beta}$ matrices. Here the $\alpha=1 \cdots 16, \beta=1-9, A, B, C$ range is displayed. Single, double and triple, but not quadruple, spin observables are shown. The entries of "phase class" $\widetilde{\Gamma}_{P L} \times \widetilde{\omega}_{P}$ do not appear-they are all quadruple spin observables.

\begin{tabular}{|c|c|c|c|c|c|c|}
\hline$\alpha \backslash \beta$ & 1 & 2 & 3 & 4 & 5 & 6 \\
\hline 1 & $\mathcal{I}$ & $\mathcal{C}_{z x^{\prime}}^{\gamma V}$ & $P_{V}$ & $\mathcal{C}_{z z^{\prime}}^{\gamma V}$ & $\mathcal{C}_{z 4}^{\gamma V}$ & $\sqrt{\frac{2}{3}} T_{21}$ \\
\hline 2 & $-\mathcal{C}_{z z^{\prime}}^{\gamma N^{\prime}}$ & $-\mathcal{C}_{z^{\prime} x^{\prime}}^{N^{\prime} V}$ & $\mathcal{C}_{z z^{\prime} y^{\prime}}^{\gamma N^{\prime} V}$ & $-\mathcal{C}_{z^{\prime} z^{\prime}}^{N^{\prime} V}$ & $-\mathcal{C}_{z^{\prime} 4}^{N^{\prime} V}$ & $\mathcal{C}_{z z^{\prime} 5}^{\gamma N^{\prime} V}$ \\
\hline \multirow[t]{2}{*}{3} & $-\mathcal{C}_{z x y^{\prime}}^{\gamma N N^{\prime}}$ & $-\mathcal{C}_{x y^{\prime} x^{\prime}}^{N N^{\prime} V}$ & $-\mathcal{C}_{y z y^{\prime}}^{\gamma N V}$ & $-\mathcal{C}_{x y^{\prime} z^{\prime}}^{N N^{\prime} V}$ & $-\mathcal{C}_{x y^{\prime} 4}^{N N^{\prime} V}$ & $-\mathcal{C}_{x y y^{\prime}}^{\gamma N V}$ \\
\hline & & $\mathcal{C}_{x z 5}^{N N^{\prime} V}$ & & $-\mathcal{C}_{x z 4}^{N N^{\prime} V}$ & $\mathcal{C}_{x z z^{\prime}}^{N N^{\prime} V}$ & $-\mathcal{C}_{y z 5}^{\gamma N V}$ \\
\hline \multirow[t]{2}{*}{4} & $-\mathcal{C}_{y y^{\prime}}^{N N^{\prime}}$ & $\mathcal{C}_{y 6}^{\gamma V}$ & $\mathcal{C}_{x y^{\prime}}^{\gamma V}$ & $\mathcal{C}_{x 4}^{\gamma V}$ & $\mathcal{C}_{y z^{\prime}}^{\gamma V}$ & $\mathcal{C}_{x 5}^{\gamma V}$ \\
\hline & & & $-\mathcal{C}_{y y^{\prime} y^{\prime}}^{N N^{\prime} V}$ & & & $-\mathcal{C}_{y y^{\prime} 5}^{N N^{\prime} V}$ \\
\hline \multirow[t]{2}{*}{5} & $-\mathcal{C}_{z z y^{\prime}}^{\gamma N N^{\prime}}$ & $\mathcal{C}_{x x 6}^{\gamma N V}$ & $-\mathcal{C}_{y x y^{\prime}}^{\gamma N V}$ & $-\mathcal{C}_{x x 4}^{\gamma N V}$ & $\mathcal{C}_{x x z^{\prime}}^{\gamma N V}$ & $\mathcal{C}_{y x 5}^{\gamma N V}$ \\
\hline & & $\mathcal{C}_{z y^{\prime} x^{\prime}}^{N N^{\prime} V}$ & & $\mathcal{C}_{z y^{\prime} z^{\prime}}^{N N^{\prime} V}$ & $\mathcal{C}_{z y^{\prime} 4}^{N N^{\prime} V}$ & \\
\hline 6 & $-\mathcal{C}_{x x^{\prime}}^{N N^{\prime}}$ & & $\mathcal{C}_{x x^{\prime} y^{\prime}}^{N N^{\prime} V}$ & & & $\mathcal{C}_{x x^{\prime} 5}^{N N^{\prime} V}$ \\
\hline \multirow[t]{2}{*}{7} & $\mathcal{C}_{z y x^{\prime}}^{\gamma N N^{\prime}}$ & $\mathcal{C}_{x z^{\prime} 6}^{\gamma N^{\prime} V}$ & $-\mathcal{C}_{y z^{\prime} y^{\prime}}^{\gamma N^{\prime} V}$ & $-\mathcal{C}_{x z^{\prime} 4}^{\gamma N^{\prime} V}$ & $\mathcal{C}_{x z^{\prime} z^{\prime}}^{\gamma N^{\prime} V}$ & $-\mathcal{C}_{y z^{\prime} 5}^{\gamma N^{\prime} V}$ \\
\hline & & $\mathcal{C}_{y x^{\prime} x^{\prime}}^{N N^{\prime} V}$ & & $\mathcal{C}_{y x^{\prime} z^{\prime}}^{N N^{\prime} V}$ & $\mathcal{C}_{y x^{\prime} 4}^{N N^{\prime} V}$ & \\
\hline 8 & $\mathcal{C}_{z x^{\prime}}^{N N^{\prime}}$ & & $-\mathcal{C}_{z x^{\prime} y^{\prime}}^{N N^{\prime} V}$ & & & $-\mathcal{C}_{z x^{\prime} 5}^{N N^{\prime} V}$ \\
\hline
\end{tabular}




\begin{tabular}{|c|c|c|c|c|c|c|}
\hline$\overline{\alpha \backslash \beta}$ & 1 & 2 & 3 & 4 & 5 & 6 \\
\hline 9 & $\mathcal{C}_{z z}^{\gamma N}$ & $-\mathcal{C}_{z x^{\prime}}^{N V}$ & $-\mathcal{C}_{z z y^{\prime}}^{\gamma N V}$ & $-\mathcal{C}_{z z^{\prime}}^{N V}$ & $-\mathcal{C}_{z 4}^{N V}$ & $-\mathcal{C}_{z z 5}^{\gamma N V}$ \\
\hline \multirow[t]{4}{*}{10} & $-T$ & & $\mathcal{C}_{y y}^{N^{\prime} V}$ & & & $\mathcal{C}_{y 5}^{N^{\prime} V}$ \\
\hline & & & $-\mathcal{C}_{y y^{\prime}}^{N V}$ & & & $-\mathcal{C}_{y 5}^{N V}$ \\
\hline & & $\mathcal{C}_{y y^{\prime} 6}^{\gamma N^{\prime} V}$ & $\mathcal{C}_{x y^{\prime} y^{\prime}}^{\gamma N^{\prime} V}$ & $-\mathcal{C}_{y y^{\prime} 4}^{\gamma N^{\prime} V}$ & $\mathcal{C}_{y y^{\prime} z^{\prime}}^{\gamma N^{\prime} V}$ & $\mathcal{C}_{x y^{\prime} 5}^{\gamma N^{\prime} V}$ \\
\hline & & $-\mathcal{C}_{z y x^{\prime}}^{\gamma N V}$ & & $-\mathcal{C}_{z y z^{\prime}}^{\gamma N V}$ & & $-\mathcal{C}_{z y 6}^{\gamma N V}$ \\
\hline 11 & $\mathcal{C}_{z x}^{\gamma N}$ & $-\mathcal{C}_{x x^{\prime}}^{N V}$ & $-\mathcal{C}_{z x y^{\prime}}^{\gamma N V}$ & $-\mathcal{C}_{x z^{\prime}}^{N V}$ & $-\mathcal{C}_{x 4}^{N V}$ & $-\mathcal{C}_{z x 5}^{\gamma N V}$ \\
\hline \multirow[t]{2}{*}{12} & $P_{N^{\prime}}$ & $\mathcal{C}_{z y^{\prime} x^{\prime}}^{\gamma N^{\prime}}$ & $-\mathcal{C}_{x y y^{\prime}}^{\gamma N V}$ & $\mathcal{C}_{z y^{\prime} z^{\prime}}^{\gamma N^{\prime} V}$ & $\mathcal{C}_{z y^{\prime} 4}^{\gamma N^{\prime} V}$ & $-\mathcal{C}_{x y 5}^{\gamma N V}$ \\
\hline & & $-\mathcal{C}_{y y 6}^{\gamma N V}$ & $\mathcal{C}_{y^{\prime} y^{\prime}}^{N^{\prime} V}$ & $\mathcal{C}_{y y 4}^{\gamma N V}$ & $-\mathcal{C}_{y y z^{\prime}}^{\gamma N V}$ & $\mathcal{C}_{y^{\prime} 5}^{N^{\prime} V}$ \\
\hline 13 & $\mathcal{C}_{x z^{\prime}}^{N N^{\prime}}$ & & $\mathcal{C}_{x z^{\prime} y^{\prime}}^{N N^{\prime} V}$ & & & $\mathcal{C}_{x z^{\prime} 5}^{N N^{\prime} V}$ \\
\hline \multirow[t]{2}{*}{14} & $\mathcal{C}_{z y z^{\prime}}^{\gamma N N^{\prime}}$ & $-\mathcal{C}_{x x^{\prime} 6}^{\gamma N^{\prime} V}$ & $\mathcal{C}_{y x^{\prime} y^{\prime}}^{\gamma N^{\prime} V}$ & $\mathcal{C}_{x x^{\prime} 4}^{\gamma N^{\prime} V}$ & $-\mathcal{C}_{x x^{\prime} z^{\prime}}^{\gamma N^{\prime} V}$ & $\mathcal{C}_{y x^{\prime} y^{\prime}}^{\gamma N^{\prime} V}$ \\
\hline & & $\mathcal{C}_{y z^{\prime} x^{\prime}}^{N N^{\prime} V}$ & & $\mathcal{C}_{y z^{\prime} z^{\prime}}^{N N^{\prime} V}$ & $\mathcal{C}_{y z^{\prime} 4}^{N N^{\prime} V}$ & \\
\hline 15 & $\mathcal{C}_{z z^{\prime}}^{N N^{\prime}}$ & & $-\mathcal{C}_{z z^{\prime} y^{\prime}}^{N N^{\prime} V}$ & & & $-\mathcal{C}_{z z^{\prime} 5}^{N N^{\prime} V}$ \\
\hline 16 & $-\mathcal{C}_{z x^{\prime}}^{\gamma N^{\prime}}$ & $-\mathcal{C}_{x^{\prime} x^{\prime}}^{N^{\prime} V}$ & $-\mathcal{C}_{z x^{\prime} y^{\prime}}^{\gamma N^{\prime} V}$ & $-\mathcal{C}_{x^{\prime} z^{\prime}}^{N^{\prime} V}$ & $-\mathcal{C}_{x^{\prime} 4}^{N^{\prime} V}$ & $-\mathcal{C}_{z x^{\prime} 5}^{\gamma N^{\prime} V}$ \\
\hline
\end{tabular}


TABLE III. Spin Observables in BHP form as products of $\Gamma^{\alpha}$, and $\omega^{\beta}$ matrices. Here the $\alpha=1 \cdots 16, \beta=1-6$ range is displayed.

\begin{tabular}{|c|c|c|c|c|c|c|}
\hline$\alpha \backslash \beta$ & 7 & 8 & 9 & $\mathrm{~A}$ & B & $\mathrm{C}$ \\
\hline 1 & $\mathcal{C}_{z 6}^{\gamma V}$ & $T_{22}$ & $T_{20}$ & $\begin{array}{c}T_{y^{\prime} y^{\prime}} \\
-\mathcal{C}_{x y y^{\prime}}^{\gamma N N^{\prime}}\end{array}$ & & \\
\hline 2 & $-\mathcal{C}_{z^{\prime} 6}^{N^{\prime} V}$ & $\mathcal{C}_{z z^{\prime} 7}^{\gamma N^{\prime} V}$ & $\mathcal{C}_{z z^{\prime} 8}^{\gamma N^{\prime} V}$ & $-\mathcal{C}_{y y x^{\prime}}^{\gamma N N^{\prime}}$ & & \\
\hline 3 & $-\mathcal{C}_{x z x^{\prime}}^{N N^{\prime} V}$ & & & $\mathcal{C}_{y z}^{\gamma N}$ & $-\mathcal{C}_{y z 7}^{\gamma N V}$ & $-\mathcal{C}_{y z 8}^{\gamma N V}$ \\
\hline 4 & $-\mathcal{C}_{y x^{\prime}}^{\gamma V}$ & $-\mathcal{C}_{y y^{\prime} 7}^{N N^{\prime} V}$ & $-\mathcal{C}_{y y^{\prime} 8}^{N N^{\prime} V}$ & $\Sigma$ & $\mathcal{C}_{x 7}^{\gamma V}$ & $\mathcal{C}_{x 8}^{\gamma V}$ \\
\hline 5 & $\begin{array}{l}\mathcal{C}_{z y^{\prime} 6}^{N N^{\prime} V} \\
-\mathcal{C}_{x x x^{\prime}}^{\gamma N V}\end{array}$ & & & $\mathcal{C}_{y x}^{\gamma N}$ & $\mathcal{C}_{y x 7}^{\gamma N V}$ & $\mathcal{C}_{y x 8}^{\gamma N V}$ \\
\hline 6 & & $\mathcal{C}_{x x^{\prime} 7}^{N N^{\prime} V}$ & $\mathcal{C}_{x x^{\prime} 7}^{N N^{\prime} V}$ & $-\mathcal{C}_{x z z^{\prime}}^{\gamma N N^{\prime}}$ & & \\
\hline 7 & $\begin{array}{l}-\mathcal{C}_{x z^{\prime} x^{\prime}}^{\gamma N^{\prime} V} \\
\mathcal{C}_{y x^{\prime} 6}^{N N^{\prime} V}\end{array}$ & & & $-\mathcal{C}_{y z^{\prime}}^{\gamma N^{\prime}}$ & $-\mathcal{C}_{y z^{\prime} 7}^{\gamma N^{\prime} V}$ & $-\mathcal{C}_{y z^{\prime} 8}^{\gamma N^{\prime} V}$ \\
\hline 8 & & $-\mathcal{C}_{z x^{\prime} 7}^{N N^{\prime} V}$ & $\begin{array}{l}-\mathcal{C}_{x x z^{\prime}}^{\gamma N N^{\prime}} \\
-\mathcal{C}_{z x^{\prime} 8}^{N N^{\prime} V}\end{array}$ & & & \\
\hline
\end{tabular}




\begin{tabular}{|c|c|c|c|c|c|c|}
\hline$\alpha \backslash \beta$ & 7 & 8 & 9 & A & B & $\mathrm{C}$ \\
\hline 9 & $-\mathcal{C}_{z 6}^{N V}$ & $-\mathcal{C}_{z z 7}^{\gamma N V}$ & $-\mathcal{C}_{z z 8}^{\gamma N V}$ & $-\mathcal{C}_{y x y^{\prime}}^{\gamma N N^{\prime}}$ & & \\
\hline \multirow[t]{2}{*}{10} & $-\mathcal{C}_{y y^{\prime} x^{\prime}}^{\gamma N^{\prime} V}$ & $\mathcal{C}_{y 7}^{N^{\prime} V}$ & $\mathcal{C}_{y 8}^{N^{\prime} V}$ & $\mathcal{C}_{x y^{\prime}}^{\gamma N^{\prime}}$ & $\mathcal{C}_{x y^{\prime} 7}^{\gamma N^{\prime} V}$ & $\mathcal{C}_{x y^{\prime} 8}^{\gamma N^{\prime} V}$ \\
\hline & & $-\mathcal{C}_{y 7}^{N V}$ & $-\mathcal{C}_{y 8}^{N V}$ & & & \\
\hline 11 & $-\mathcal{C}_{x 6}^{N V}$ & $-\mathcal{C}_{z x 7}^{\gamma N V}$ & $-\mathcal{C}_{z x 8}^{\gamma N V}$ & $-\mathcal{C}_{y z y^{\prime}}^{\gamma N N^{\prime}}$ & & \\
\hline \multirow[t]{2}{*}{12} & $\mathcal{C}_{z y^{\prime} 6}^{\gamma N^{\prime} V}$ & $\mathcal{C}_{y^{\prime} 7}^{N^{\prime} V}$ & $\mathcal{C}_{y^{\prime} 8}^{N^{\prime} V}$ & $-\mathcal{C}_{x y}^{\gamma N}$ & $-\mathcal{C}_{x y 7}^{\gamma N V}$ & $-\mathcal{C}_{x y 8}^{\gamma N V}$ \\
\hline & $\mathcal{C}_{y y x^{\prime}}^{\gamma N V}$ & & & & & \\
\hline 13 & & $-\mathcal{C}_{x z^{\prime} 7}^{N N^{\prime} V}$ & $-\mathcal{C}_{x z^{\prime} 8}^{N N^{\prime} V}$ & $\mathcal{C}_{x z x^{\prime}}^{\gamma N N^{\prime}}$ & & \\
\hline \multirow[t]{2}{*}{14} & $\mathcal{C}_{x x^{\prime} x^{\prime}}^{\gamma N^{\prime} V}$ & & & $\mathcal{C}_{y x^{\prime}}^{\gamma N^{\prime}}$ & $\mathcal{C}_{y x^{\prime} 7}^{\gamma N^{\prime} V}$ & $\mathcal{C}_{y x^{\prime} 8}^{\gamma N^{\prime} V}$ \\
\hline & $\mathcal{C}_{y z^{\prime} 6}^{N N^{\prime} V}$ & & & & & \\
\hline \multirow[t]{2}{*}{15} & & $-\mathcal{C}_{z z^{\prime} 7}^{N N^{\prime} V}$ & $-\mathcal{C}_{z z^{\prime} 8}^{N N^{\prime} V}$ & & & \\
\hline & & & $\mathcal{C}_{x x x^{\prime}}^{\gamma N N^{\prime}}$ & & & \\
\hline 16 & $-\mathcal{C}_{x^{\prime} 6}^{N^{\prime} V}$ & $-\mathcal{C}_{z x^{\prime} 7}^{\gamma N^{\prime} V}$ & $-\mathcal{C}_{z x^{\prime} 8}^{\gamma N^{\prime} V}$ & $\mathcal{C}_{y y z^{\prime}}^{\gamma N N^{\prime}}$ & & \\
\hline
\end{tabular}


Fig. 2: Pichowsky et al. "Polarization observables ..."

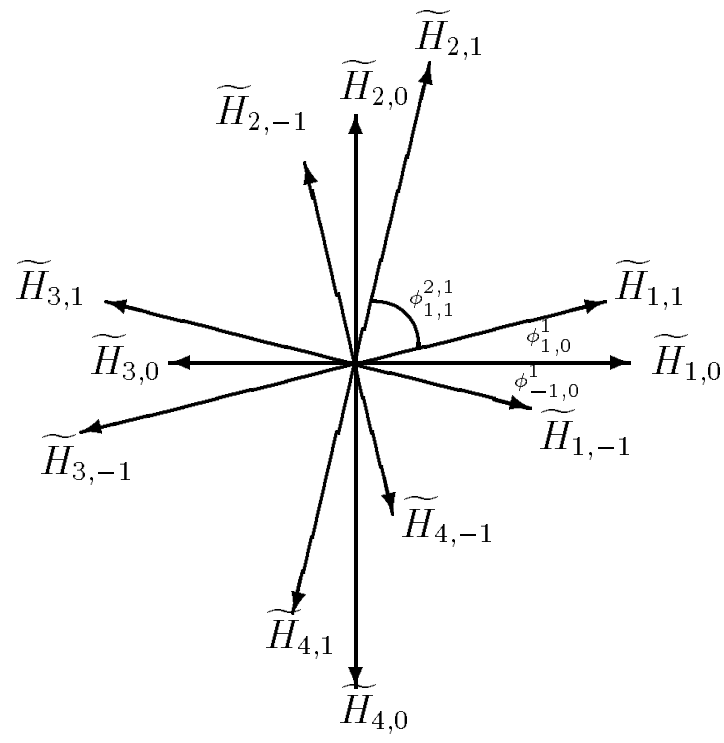


Fig. 3: Pichowsky et al., "Polarization observables ..."

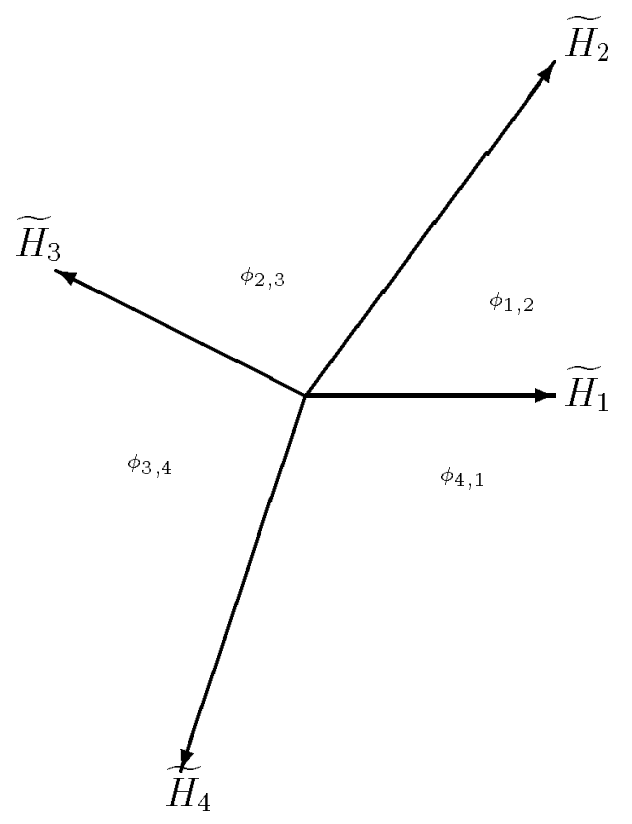


Fig. 1: Pichowsky et al. "Polarization observables in vector mesonphotoproduction"

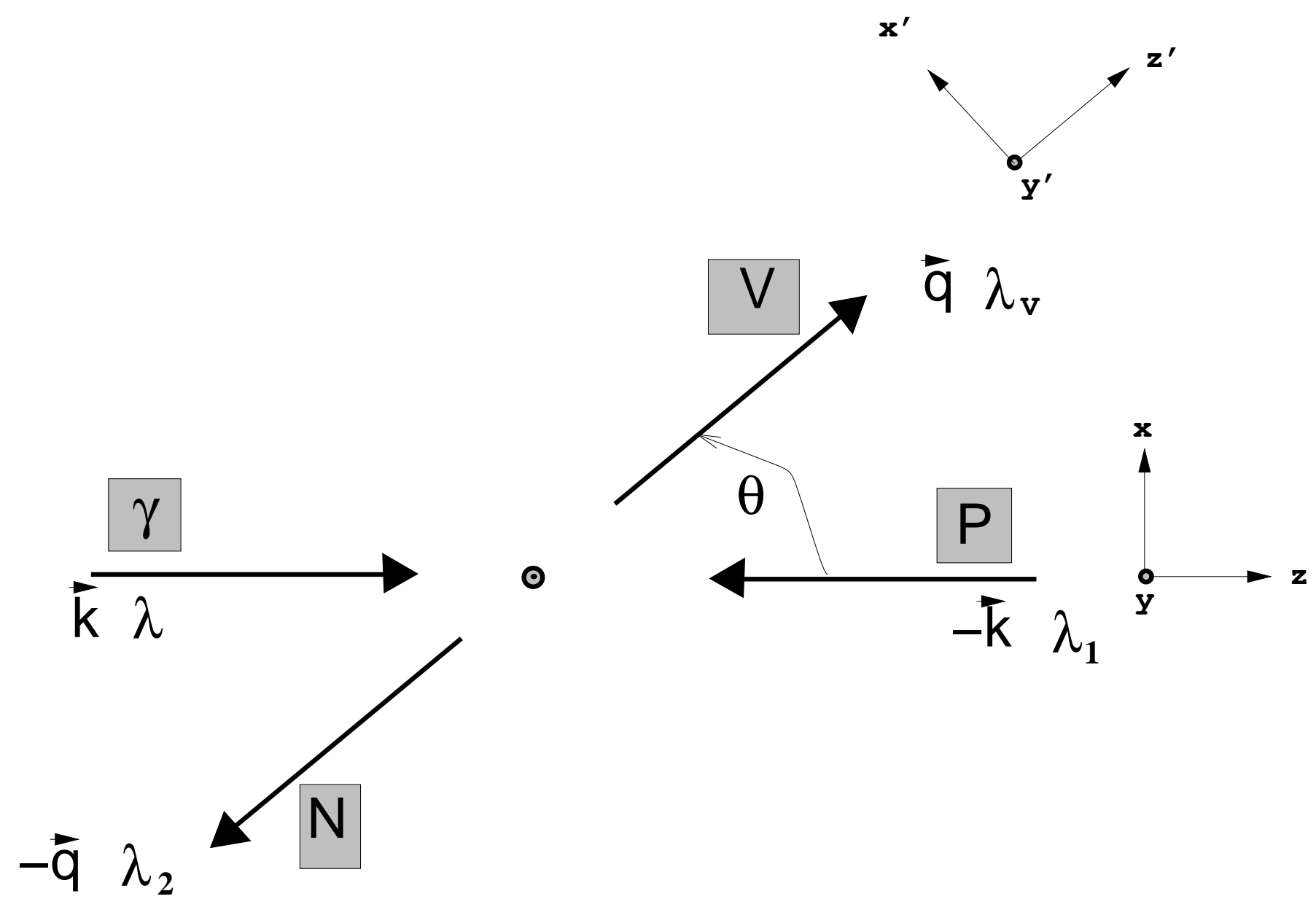

